\title{
REVISIÓN TAXONÓMICA DEL COMPLEJO ACUTIFOLIAE DE QUERCUS (FAGACEAE) CON ÉNFASIS EN SU REPRESENTACIÓN EN MÉXICO
}

\author{
Silvia Romero RANGel \\ Universidad Nacional Autónoma de México, Facultad de Estudios Superiores \\ Iztacala, Laboratorio de Ecología y Taxonomía de Árboles y Arbustos, \\ Apdo. postal 314, 54090 Tlalnepantla, Estado de México, México
}

\section{RESUMEN}

Se revisa taxonómicamente la serie Acutifoliae del género Quercus (Fagaceae) que consta de diez especies: Quercus acutifolia Née, Q. albocincta Trel., Q. brenesii Trel., Q. canbyi Trel., Q. conspersa Benth., Q. cortesii Liebm., Q. furfuracea Liebm., Q. skinneri Benth., Q. uxoris McVaugh y Q. xalapensis Humb. \& Bonpl. El grupo se distribuye desde el sureste de Estados Unidos de América hasta Costa Rica; cinco especies son endémicas de México.

Palabras clave: Acutifoliae, encinos, Fagaceae, Quercus, taxonomía.

\section{ABSTRACT}

Quercus series Acutifoliae is revised. The series is composed of ten species: Quercus acutifolia Née, Q. albocincta Trel., Q. brenesii Trel., Q. canbyi Trel., Q. conspersa Benth., Q. cortesii Liebm., Q. furfuracea Liebm., Q. skinneri Benth., Q. uxoris McVaugh and Q. xalapensis Humb. \& Bonpl. The series is distributed from southeastern United States of America to Costa Rica; five species are endemic to Mexico.

Key words: Acutifoliae, Fagaceae, oaks, Quercus, taxonomy.

\section{INTRODUCCIÓN}

El género Quercus L. ha estado sujeto a varias clasificaciones taxonómicas, entre ellas destaca la de Trelease (1924) para los encinos americanos, quien 
propone para este continente la división en 131 series agrupadas en los subgéneros Protobalanus Trel., Leucobalanus Engelm. y Erythrobalanus (Spach) Endl.

Camus (1938), en un estudio monográfico sobre el género a escala mundial, divide a Quercus en dos subgéneros: Cyclobalanopsis (Oersted) Schneider y Euquercus (Hickel et Camus) Camus, fraccionando a este último en seis secciones: Cerris Loudon, Mesobalanus Camus, Lepidobalanus Endl., Macrobalanus (Oerst.) Schwarz, Protobalanus Trel. y Erythrobalanus (Spach) Schwarz.

Con base en análisis filogenéticos recientes, Nixon (1993) reconoce dos subgéneros: Quercus y Cyclobalanopsis; el primero se divide en tres secciones Lobatae, Protobalanus y Quercus.

El trabajo de Trelease (1924), a pesar de ser el más antiguo de los citados, es el único que propone la clasificación de los encinos en series, aspecto que permite definir conjuntos más reducidos de especies relacionadas, por lo menos morfológica y geográficamente. Müller (1942a) en aquel tiempo menciona al respecto, que es el mejor esfuerzo para agrupar a los componentes de Quercus en rangos infragenéricos. De hecho se puede decir que ha sido el único intento de esta naturaleza. Sin embargo, no se ha confirmado que las series sean monofiléticas y es muy probable que éste no sea el caso para la gran mayoría de ellas.

Mientras que Trelease (1924) ubica a la serie Acutifoliae, una de las más grandes de encinos rojos, en el subgénero Erythrobalanus; Nixon (1993) indica que los encinos rojos deben tratarse como sección Lobatae. Trelease (1924) la define con las siguientes características: porte arbóreo, ramillas glabras; yemas ovoidefusiformes; hojas lanceoladas, glabras, con dientes aristados; pecíolos largos; frutos anuales o bianuales sobre pedúnculos muy cortos, de tamaño mediano a grande, usualmente tomentosos, con escamas adpresas. Para el mencionado autor el grupo se integra con once especies, además de nueve formas y tres variedades.

Posteriormente Müller (1936a, 1936b, 1942a, 1942b, 1951) adiciona varias especies y reconoce como sinónimos de la serie Acutifoliae a las series Grandes Trel., Huitamalcanae Trel., Brenesieae Trel., Skinneriae Trel. y Albocinctae Trel. En estudios siguientes, Martínez (1954) considera como sinónimos de Q. conspersa Benth. a Q. correpta Trel., Q. conspersa var. ovatifolia Trel. y Q. conspersa var. caudata Trel. De acuerdo con Trelease (1924), Q. correpta Trel. pertenece a la serie Guatimalenses.

Müller y McVaugh (1972) estiman que la serie Acutifoliae está formada por 15 o más especies (sin mencionar todos los nombres de ellas), e indican que es necesaria su revisión. En este mismo trabajo se describe como especie nueva a Quercus uxoris McVaugh, la que se incluye en la serie Acutifoliae (Cuadro 1). 
Cuadro 1. Especies del complejo Acutifoliae reconocidas por distintos autores, indicando las correspondencias con el presente trabajo.

\begin{tabular}{|c|c|c|c|c|c|c|c|c|}
\hline Romero, este trabajo & Trelease, 1924 & Müller, 1936a & Müller, 1936b & Müller, 1942a & Müller, 1942b & Müller, 1951 & Mártinez, 1954 & Müller \& McVaugh, 1972 \\
\hline Q. acutifolia Née & $\begin{array}{l}\text { Q. acutifolia Née } \\
\text { Q. conspersa f. caudata Trel. } \\
\text { Q. conspersa f. ovatifolia Trel. } \\
\text { Q. grahamii Benth. } \\
\text { Q. grahamii var. nelsonii Trel. } \\
\text { Q. grahamii f. brevipes Trel. } \\
\text { Q. toniaguiae Trel. }\end{array}$ & Q. grahamii Benth. & & $\begin{array}{l}\text { Q. acutifolia Née } \\
\text { Q. anglohondurensis Müll. } \\
\text { Q. monserratensis Müll. } \\
\text { Q. tenuiaristata Trel. }\end{array}$ & & & Q. acutifolia Née & Q. acutifolia Née \\
\hline Q. albocincta Trel. & Q. albocincta Trel. & & & Q. albocincta Trel. & & & & \\
\hline Q. brenesii Trel. & Q. brenesii Trel. & & & Q. brenesii Trel. & & & & \\
\hline Q. canbyi Trel. & $\begin{array}{l}\text { Q. canbyi Trel. } \\
\text { Q. canbyi f. adscendens Trel. } \\
\text { Q. canbyi f. berlandieri Trel. } \\
\text { Q. karwinskii Trel. }\end{array}$ & Q. graciliformis Müll. & $\begin{array}{l}\text { Q. alamarensis Müll. } \\
\text { Q. canbyi f. attenuata Müll. } \\
\text { Q. canbyi f. karwinskii Müll. } \\
\text { Q. canbyi f. pedunculata Müll. } \\
\text { Q. canbyi f. setacea Müll. } \\
\text { Q. graciliramis Müll. }\end{array}$ & & & Q. gracilliformis Müll. & & \\
\hline Q. conspersa Benth. & $\begin{array}{l}\text { Q. conspersa Benth. } \\
\text { Q. correpta Trel. } \\
\text { Q. uruapanensis Trel. }\end{array}$ & & & Q. conspersa Benth. & & & Q. conspersa Benth. & Q. conspersa Benth. \\
\hline Q. cortesii Liebm. & Q. cortesii Liebm. & & & Q. cortesii Liebm. & & & & \\
\hline Q. furfuracea Liebm. & Q. furfuracea Liebm. & & & & & & & \\
\hline Q. skinneri Benth. & $\begin{array}{l}\text { Q. skinneri Benth. } \\
\text { Q. chiapasensis Trel. } \\
\text { Q. chiapasensis f. cuneifolia Trel. } \\
\text { Q. chiapasensis f. falcilobata Trel. } \\
\text { Q. chiapasensis f. flagellata Trel. } \\
\text { Q. chiapasensis f. longipes Trel. } \\
\text { Q. chiapasensis f. petiolata Trel. } \\
\text { Q. chiapasensis f. subcuneata Trel. } \\
\text { Q. grandis Liebm. } \\
\text { Q grandis var. tenuipes Trel. } \\
\text { Q. salvadorensis Trel. } \\
\text { Q. trichodonta Trel. }\end{array}$ & & & Q. skinneri Benth. & & & & \\
\hline Q. uxoris McVaugh & & & & & & & & Q. uxoris McVaugh \\
\hline Q. xalapensis Humb. \& Bonpl. & $\begin{array}{l}\text { Q. xalapensis Humb. \& Bonpl. } \\
\text { Q. candolleana Trel. } \\
\text { Q . huitamalcana Trel. } \\
\text { Q. xalapensis f. jalapae Trel. } \\
\text { Q. xalapensis f. surculina Trel. } \\
\text { Q. sartorii Liebm. } \\
\text { Q. sartorii f. magna Liebm. } \\
\text { Q. vexans Trel. }\end{array}$ & $\begin{array}{l}\text { Q. cupreata Trel. \& Müll. } \\
\text { Q. runcinatifolia f. alata Trel. } \\
\text { \& Müll. }\end{array}$ & $\begin{array}{l}\text { Q. cupreata f. brachystachys Müll. } \\
\text { Q. cupreata f. serrata Trel. \& Müll. } \\
\text { Q. sartorii Liebm. } \\
\text { Q. tenuiloba Müll. } \\
\text { Q. tenuiloba f. hirsuta Müll. } \\
\text { Q. vexans Trel. }\end{array}$ & $\begin{array}{l}\text { Q. huitamalcana Trel. } \\
\text { Q. paxtalensis Müll. }\end{array}$ & Q. sierramadrensis Müll. & & & \\
\hline
\end{tabular}


El complejo en estudio, considerando las contribuciones anteriores, está integrado por 57 nombres de taxa, muchos de ellos ya reducidos a sinónimos, incluyendo especies, variedades y formas, que muestran similitud morfológica con Quercus acutifolia. En este trabajo se reconocen diez especies, que no son exactamente las incluidas en la serie por Trelease (1924).

\section{MÉTODOS}

Se revisaron ejemplares depositados en los herbarios A, B, BH, C, DPU, ENCB, G, ILL, IZTA, K, M, MA, MBG, MEXU, MICH, MO, NY, TEX y US. Las colecciones examinadas sumaron aproximadamente 650 ejemplares, entre los cuales se incluyen tipos de 45 nombres de taxa involucrados con la serie Acutifoliae. Los especímenes tipo que no pudieron verse fueron estudiados a través de sus fotografías publicadas en las obras de Trelease (1924) y Müller (1942a).

Se realizaron salidas al campo para definir la variación de algunos caracteres morfológicos a nivel de poblaciones y recolectar material. Los ejemplares correspondientes se depositaron en los herbarios del Instituto de Biología de la Universidad Nacional Autónoma de México (MEXU), de la Escuela Nacional de Ciencias Biológicas del Instituto Politécnico Nacional (ENCB) y en el de la Facultad de Estudios Superiores Iztacala (IZTA).

La parte taxonómica incluye la clave de identificación y las descripciones de los taxa reconocidos, acompañadas de la información nomenclatural correspondiente. Los tipos de tricomas se designan de acuerdo con el criterio de Jones (1986). Además, se incluyen las listas de los ejemplares consultados y su ubicación en los herbarios, así como ilustraciones de las especies reconocidas.

\section{ESTUDIO TAXONÓMICO}

Clave para la identificación de las especies de la serie Acutifoliae

1. Hojas maduras sin dientes en los márgenes; con frecuencia el envés de las hojas con abundantes tricomas glandulares de color ámbar. Árboles del centro y sureste de México, así como de Guatemala

Q. conspersa

1. Hojas maduras con dientes en los márgenes. 
2. Ramillas y hojas densamente pubescentes, principalmente en el envés de las hojas; la pubescencia persiste por un año o más.

3. Hojas de 8 a $30 \mathrm{~cm}$ de largo con 9 a 18 dientes aristados en los márgenes de cada lado, con indumento amarillento. Árboles del centro-oeste y sureste

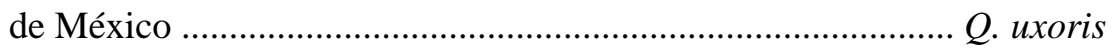

3. Hojas de 4.5 a $10 \mathrm{~cm}$ de largo con 3 a 10 dientes aristados en los márgenes de cada lado, con indumento canescente. Árboles del centro de México .... Q. furfuracea

2. Ramillas y hojas glabras o casi glabras, algunas veces se encuentran tricomas en las axilas de las nervaduras principales o muy dispersos en el envés de las hojas.

4. Hojas maduras angostamente elípticas o angostamente lanceoladas.

5. Hojas con los dientes ubicados en los márgenes de la mitad superior de la lámina.

6. Dientes de los márgenes de la hoja divergentes, nervaduras 7-11 en cada lado; envés de las hojas con escasos tricomas sobre la nervadura primaria de la lámina .................................. Q. brenesii

6. Dientes de los márgenes de la hoja no divergentes, nervaduras 10-15 en cada lado; envés de las hojas con tricomas en las axilas de las nervaduras principales Q. cortesii

5. Hojas con los dientes ubicados en todo el margen o al menos en las 2/3 partes superiores de la lámina

7. Hojas con 3 a 5 dientes en los márgenes de cada lado, éstos de más de $15 \mathrm{~mm}$ de largo. Árboles del sureste de Estados Unidos de América y noreste de México Q. canbyi

7. Hojas con 6-15 dientes en los márgenes de cada lado, éstos hasta de 8 mm de largo. Árboles del centro-oeste, sur y sureste de México ..... Q. acutifolia

4. Hojas maduras elípticas u ovadas.

8. Frutos de 20 a $50 \mathrm{~mm}$ de diámetro y de 15 a $50 \mathrm{~mm}$ de largo. Árboles del este y sur de México y Guatemala Q. skinneri

8. Frutos de $5-10 \mathrm{~mm}$ de diámetro y de 6 a $20 \mathrm{~mm}$ de largo.

9. Márgenes de las hojas con dientes de 3-5 mm de largo. Árboles del noroeste de México Q. albocincta

9. Márgenes de las hojas con dientes de 0.5-2 mm de largo. Árboles del noreste, este, oeste, centro y sur de México. 
10. Venas secundarias formando ángulos de divergencia uniformes y las venas intersecundarias ubicándose en la parte media de la lámina; yemas de 2-6 mm de largo. Árboles del centro y suroeste de México ........................................... Q. acutifolia

10. Venas secundarias formando ángulos de divergencia que disminuyen hacia el ápice y las venas intersecundarias ubicándose en el ápice y la base de la lámina; yemas de 1-3 $\mathrm{mm}$ de largo. Árboles del noreste, este y centro de México Q. xalapensis

Quercus acutifolia Née, An. Ci. Nat. 3: 267. 1801. Tipo: México, Guerrero, cerca de Tixtla, Née s. n. (holotipo en MA!). (Fig. 1).

Quercus anglohondurensis Müll., Misc. Publication 477, U.S. Dept. of Agriculture. 76. Lám. 114. 1942. Tipo: Belice, San Agustín, Lundell 6615 (holotipo en MICH, isotipo en MICH!).

Quercus conspersa f. caudata Trel., Mem. Nat. Acad. Sci. 20: 192. Lám. 389. 1924.

Tipo: Guatemala, Lehmann 1320 (holotipo en B!).

Quercus conspersa f. ovatifolia Trel., Mem. Nat. Acad. Sci. 20: 192. Lám. 389. 1924.

Tipo: Guatemala, Warscewicz 28 (holotipo en G).

Quercus grahamii Benth., Plant. Hartweg., p. 57. 1840. Tipo: México, sin localidad, Graham 326 (holotipo en K!).

Quercus grahamii var. nelsonii Trel., Mem. Nat. Acad. Sci. 20: 190. 1924. Tipo: México, Oaxaca, S. Miguel Albarrados, Nelson 533 (holotipo en US, isotipo en US!).

Quercus grahamii f. brevipes Trel., Mem. Nat. Acad. Sci. 20: 190. 1924. Tipo: México, Veracruz, Puente Colorado, Cuesta de Aculcingo, Liebmann 3437 (holotipo en $\mathrm{K}$ !).

Quercus monserratensis Müll., Misc. Publication 477, U.S. Dept. of Agriculture. pp. 71-72. 1942. Tipo: México, Chiapas, near Hac. Monserrate, Purpus 10091 (holotipo en A).

Quercus tenuiaristata Trel., Field Mus. Bot. Ser. 17: 358. 1938. Tipo: Honduras, Dept. Comayagua, Yuncker, Dawson \& Youse 6204 (holotipo en DPU).

Quercus tonaguiae Trel., Mem. Nat. Acad. Sci. 20: 190. 1924. Tipo: México, Oaxaca, Tonaguia, Galeotti s. n. (holotipo en B). 
Romero Rangel: Revisión taxonómica del complejo Acutifoliae de Quercus

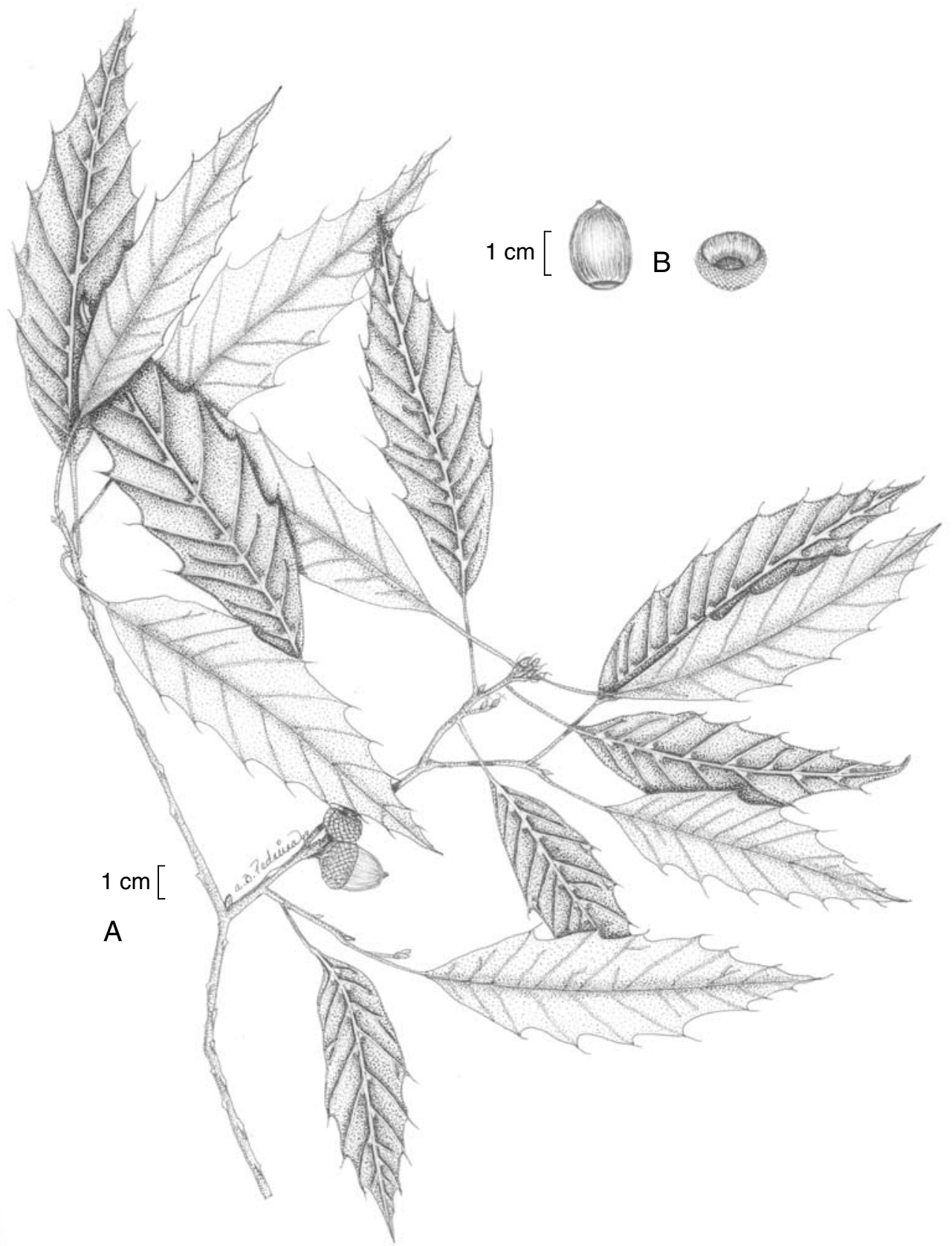

Fig. 1. Quercus acutifolia. A. rama fértil con hojas y frutos (Rzedowski 18084, ENCB); B. fruto y cúpula (Judziewicz 4301, ENCB). 
Árbol de 3-30 m de alto y con tronco de 0.25-1 m de diámetro; corteza oscura y agrietada, ramillas de 1-2 (-3) mm de diámetro, glabrescentes, el indumento perdura una temporada, obscuras, lustrosas, con lenticelas claras, de 0.5-1 mm de largo; yemas ovoides con el ápice agudo, de 2-6 mm de largo, con las escamas pubescentes, de color castaño; estípulas linear-oblanceoladas, de 7$12 \mathrm{~mm}$ de largo por 1-2 mm de ancho, deciduas antes de la madurez de las hojas; hojas jóvenes pubescentes, haz con tricomas fasciculados estipitados cortos sobre la lámina, tricomas fasciculados estipitados largos sobre la nervadura primaria y tricomas glandulares abundantes, envés con tricomas fasciculados estipitados cortos distribuidos en la lámina, estrellados largos en las axilas de las nervaduras y tricomas glandulares de color ámbar abundantes; hojas maduras por lo general angostamente elípticas, a veces elípticas, de (6-) 8-15 (-24) cm de largo por (1-) 2-6.5 (-8.5) cm de ancho, gruesas o delgadas, ápice atenuado o agudo, márgenes con dientes cortos, aristados, distribuidos desde la base hasta el ápice, o en las dos terceras partes superiores de la lámina, dientes 6-12 (-15) en cada lado del margen de la lámina, ascendentes, triangulares, hasta de $8 \mathrm{~mm}$ de largo, aristas de 1-7 mm de largo; nervaduras secundarias 5-12 (-16) en cada lado de la lámina de la hoja, pasando directamente hasta los dientes; haz algo lustroso, las nervaduras secundarias y terciarias forman un evidente retículo pálido, casi glabro, con tricomas en la base de la nervadura primaria; envés más pálido que el haz, nervaduras convexas, casi glabro, con frecuencia se observan tricomas fasciculados estipitados cortos en la base de la nervadura primaria, tricomas fasciculados estipitados largos en las axilas de la nervaduras y a menudo también se observan tricomas glandulares de color ámbar cerca de la nervadura primaria, epidermis lisa; pecíolos de (5-) 10-25 (-30) mm de largo por 0.5-1.5 mm de ancho, glabros o con algunos tricomas fasciculados estipitados en la base; amentos masculinos de 7-13 cm de largo, con 10 a 35 flores o más, perianto sésil, muy pubescente, de 2-3 mm de diámetro, anteras 5 , glabras, elipsoidales, de 1-2 mm de largo, filamentos de 1-2 mm de largo; amentos femeninos de 5-20 mm de largo, con 1 a 5 flores; fruto anual o bianual, solitario o en pares, pedúnculo de 2.5-4 mm de diámetro; cúpula hemisférica o a veces turbinada, de 920 mm de diámetro, de 7-15 mm de alto, borde recto, a veces enrollado, escamas de color castaño, pubescentes, ápices obtusos, adpresos; bellota ovoide, de 11-17 mm de largo por 9-14 mm de diámetro, de color castaño pálido, incluida en la cúpula de un tercio a un medio de su largo.

Distribución y ecología. Se le encuentra en México, en los estados de Chiapas, Guerrero, Jalisco, México, Michoacán, Oaxaca y Puebla, así como en Belice, 
Guatemala y Honduras; habitando en bosque de encino, bosque de pino-encino, bosque mesófilo de montaña y bosque tropical caducifolio, entre los 600 y $2440 \mathrm{~m}$ s.n.m. Florece de diciembre a abril y fructifica de junio a febrero.

Nombres vulgares. Encino blanco, encino de asta, encino laurelillo, encino rojo, encino saucillo, encino sencillo, encino teposcohuite.

Ejemplares examinados. MÉXICO. Chiapas: Bochil, $2 \mathrm{~km}$ al E de Bochil sobre la carretera de Jilotol, Rzedowski 33171 (ENCB); Motozintla de Mendoza, Tolimón 38 km de Huixtla, Breedlove 30946 (ENCB); San Bartolomé de los Llanos, cerro de Santolón, Weber 2099 (ENCB); Villa Corzo, cerro Tres Picos near cerro Bola along a logging road southwest of Colonia Agrónomos Mexicanos, Breedlove 24091 (ENCB); municipio indefinido, a $3 \mathrm{~km}$ de la Col. Benito Juárez en el cerro Rincón Novillo y Relumbra el Agua, Chavelas ES-4391 (ENCB). Guerrero: Alcozauca, km 4 sobre el camino San José Laguna a Alcozauca, Lorea 2936 (MEXU); Alcozauca, Martínez 522 (MEXU); paraje La Laguna, Ortiz 2162 (MEXU); 1.2 km al SE de Ixcuinatoyac, Zúñiga 18 (ENCB); Chichihualco, Heliodoro Castilllo, Filo Zancudo, Palacios 19 (MEXU); $1 \mathrm{~km}$ norte del altar de la Virgen, carretera a Tlacotepec a 8 $\mathrm{km}$ al S de Tlacote, Valencia 361 (ENCB); 7-8 km al S de Tlacotepec, carretera Filo de Caballo-Tlacotepec, Valencia 360 (MEXU); Ojo de Agua, Filo de Caballo, May Nah AM-229 (MEXU); Chichihualco, $25 \mathrm{~km}$ al SW de Xochipala, camino a Filo de Caballo, Martínez 731 (MEXU); Cruz de Ocote, sobre el camino de Xochipala al aserradero Agua Fría, Rzedowski 282 (ENCB); Chilapa, 14 km al NE de Tixtla y $2 \mathrm{~km}$ al noroeste de La Estacada, Lorea 997 (ENCB); Chilpancingo, Omiltemi, Hernández 26 (ENCB, MEXU); entre Chilpancingo y Tixtla, Müller 9162 (ENCB); Puentecillas, García 2290 (ENCB); between Chilpancingo and Tixtla, Müller 9160 (ENCB); alrededores de Chilpancingo, cerca del río Mezcala, Rojas \& Romero 4770 (IZTA); 8 km al W de Amojileca, Rodríguez 51 (MEXU); cerro Alquitrán, cerca de Mazatlán, Rzedowski 23695 (ENCB); 5 km al E de Omiltemi, Rzedowski 15980 (ENCB); camino entre el cerro del Toro y el cerro de la Vaca, Verduzco 192 (MEXU); Mazatlán, falda E del cerro El Alquitrán, Kruse 1839 (ENCB); distrito de Mina, Manchón, Hinton 9216 (MEXU); Mochitlán, Acahuizotla, Valencia 140 (MEXU); Agua de Obispo por el camino al rancho de los Carreto, Valencia 283 (MEXU); Coyuca, cerro de la Mesa, García 2181 (ENCB); San Luis Acatlán, 4 km al N de San Luis Acatlán, Valencia 732 (MEXU); Tixtla de Guerrero, Vilchis s. n. (MEXU); NE de Tlacoapa, Calzada 17269, 17284 (MEXU); Tlacotepec, cercanías de Tlacotepec, Paray 2832 (MEXU); Xaltianguis, mesa below Cajetito on cerro W 
of Xaltianguis, ca. 50 km N of Acapulco, Müller 9194 (MEXU); Zumpango, 7 km al sur de Miraval, Gónzalez-Medrano 6237 (MEXU); Municipio indefinido, entre Chilpancingo y Tixtla, Müller 9162 (MEXU). Jalisco: Cuautitlán, 8-9 km al SE del Durazno bajando la cumbre, Rosales 1926 (MEXU); Cuautitlán, km 27 al S de El Chante camino a las Capillas, Villarreal 2470 (ENCB); El Chante, at fork in road 2 $\mathrm{km}$ below and $\mathrm{N}$ of La Cumbre, Iltis 2326 (MEXU); from El Chante to El Guisar, Breedlove 61767 (MEXU); Jilotlán de Dolores, $10 \mathrm{~km}$ al S de Tecalitlán, Breedlove 64251 (MEXU); Talpa, La Cuesta, 15 km al sur de Talpa, Rzedowski 15209 (ENCB); Pihuamo, sin localidad, Martínez 63 (MEXU); Pihuamo, sin localidad, Martínez 164a (ENCB); Autlán, Rincón de Manantlán a Zarzamora, 2 km al E de Las Joyas, Iltis 1321 (MEXU); Zapotitlán, Cruz del Fresno, predio de la Hda. de San Antonio, Mancera MO-321 (MEXU). México: Valle de Bravo, Rancho Rincón Grande Tiloxtoc, Müller 9082 (MEXU); Arroyo forest, rancho Rincon Grande, Müller 9082 (MEXU); 8 a km al S de Valle de Bravo, Cerro Gordo, Müller 9088 (MEXU). Michoacán: Ciudad Hidalgo, Las Presitas, Unidad forestal "San José" 2514 (MEXU); Coalcomán de Matamoros, Puerto del Pinabete, Madrigal 3238 (ENCB); El Álamo, $35 \mathrm{~km}$ al E de Morelia sobre la carretera Zitácuaro (km 278), Rzedowski 18366 (ENCB); Villa Madero, Los Guayabos, Madrigal 3459 (ENCB, MEXU). Oaxaca: Coyula, by Mex. 190.6 mi. NW El Coyula, Kral 25306 (ENCB); Flasuaco, río de Tablas, MacDougall 2542 (MEXU); Huajuapan, 21 km al noroeste de Sinaxtla, $13 \mathrm{~km}$ al noroeste de Yanhuitlán, Espejo 3157 (MEXU); Juquila, 10 km al N de Lachao, km 170 carretera Oaxaca-Puerto Escondido, Rzedowski 19627 (ENCB); Macuiltianguis, camino al río Yesi-Yoo, Jarillo 41 (ENCB); Nacaltepec, al N de Jayacatlan, Breedlove 35982 (MEXU); Nochixtlán, 18 km SE of Nochixtlán in the Mixteca Alta, Müller 9403 (ENCB); Quiatoni, camino a Agua Canoa, Flores 1293 (MEXU); Quiotepec, Dpto. Ixtlán, W de La Soledad Tectitlán, Gereau 1989 (ENCB); Santiago Tejupan, 11 km SE de Tejupan, Müller 9396 (ENCB), 9397 (MEXU); Yautepec, 5 km al W de El Camarón, Acosta 753 (ENCB); $13 \mathrm{~km}$ E de El Camarón, Müller 9421 (MEXU); Zimatlán, Sn. Bernardo Mixtepec, López 688 (ENCB); Carrizal-Yolox, Miranda 1053 (MEXU); 69 miles NW of Tehuantepec, Lathrop 6722 (MEXU). Puebla: Municipio indefinido, km 147 de la carretera México-Tehuantepec, Ávila s. n. (MEXU).

Quercus acutifolia se caracteriza por sus hojas maduras angostamente elípticas, con el ápice atenuado o agudo, márgenes con dientes aristados distribuidos desde la base hasta el ápice, o en las dos terceras partes superiores, y en las cuales con frecuencia se observan tricomas glandulares de color ámbar. 
Esta especie se ha confundido con Quercus conspersa; sin embargo, en el presente estudio se considera que los individuos y ejemplares que poseen márgenes foliares dentado-aristados corresponden a $Q$. acutifolia y los que presentan hojas enteras, pertenecen a $Q$. conspersa (Romero R. et al., 2000a), lo cual corresponde con lo observado por González-Villarreal en Jalisco (1986). Es importante mencionar que las plantas de Q. acutifolia presentan variabilidad morfológica en sus hojas, en relación con la edad y posición de las ramas. Es decir, las hojas de individuos jóvenes, las ramas de tocones y las ramas inferiores de los tallos principales con mucha frecuencia llevan hojas ovadas, de mayor tamaño y más coriáceas que las de árboles maduros. Considerando lo anterior se determinaron como sinónimos de $Q$. acutifolia a Q. anglohondurensis Müll., Q. conspersa f. caudata Trel., Q. conspersa f. ovatifolia Trel., Q. graciliformis Müll., Q. grahamii Benth., Q. grahamii var. nelsonii Trel., Q. grahamii f. brevipes Trel., Q. monserratensis Müll., Q. tenuiaristata Trel. y Q. tonaguiae Trel. (Cuadro 1).

Quercus acutifolia también se ha confundido con Q. sartorii, la cual se considera en este trabajo sinónimo de $Q$. xalapensis; esta última especie habita en el noreste, este, centro y sureste de México y se reconoce porque las láminas de sus hojas tienen forma elíptica u ovada, el ángulo de divergencia de las venas secundarias disminuye hacia el ápice y las venas intersecundarias se ubican en el ápice y la base de la lámina. En Q. acutifolia, planta que habita en el centro y sureste de México, las láminas de las hojas tienen forma angostamente elíptica, las venas secundarias forman ángulos de divergencia uniformes y las venas intersecundarias se ubican en la parte media de la lámina. En árboles que se han cortado y en individuos jóvenes se observan hojas con formas variables, lo que provoca confusión en la determinación de los ejemplares (Romero R., 2001).

Quercus albocincta Trel., Mem. Nat. Acad. Sci. 20: 193. Lám. 394. 1924. Tipo: México, Sonora, Sierra de Álamos, Rose, Standley \& Russell 12787 (sintipos en NY y US!). (Fig. 2).

Árbol de 3-15 m de alto y con tronco de 20-50 cm de diámetro; corteza de color castaño, ramillas de 2-4 mm de diámetro, canescentes y glabrescentes, rojizas a oscuras, con abundantes lenticelas de color claro, menores de $1 \mathrm{~mm}$ de largo; yemas ovoides, rojizas, con el ápice agudo, de 2-6 mm de largo, con las escamas pubescentes; estípulas lineares, muy pronto deciduas, pubescentes, rojizas; hojas jóvenes pubescentes, haz con tricomas fasciculados estipitados cortos distribuidos sobre la lámina y tricomas glandulares escasos, envés con tricomas fasciculados 


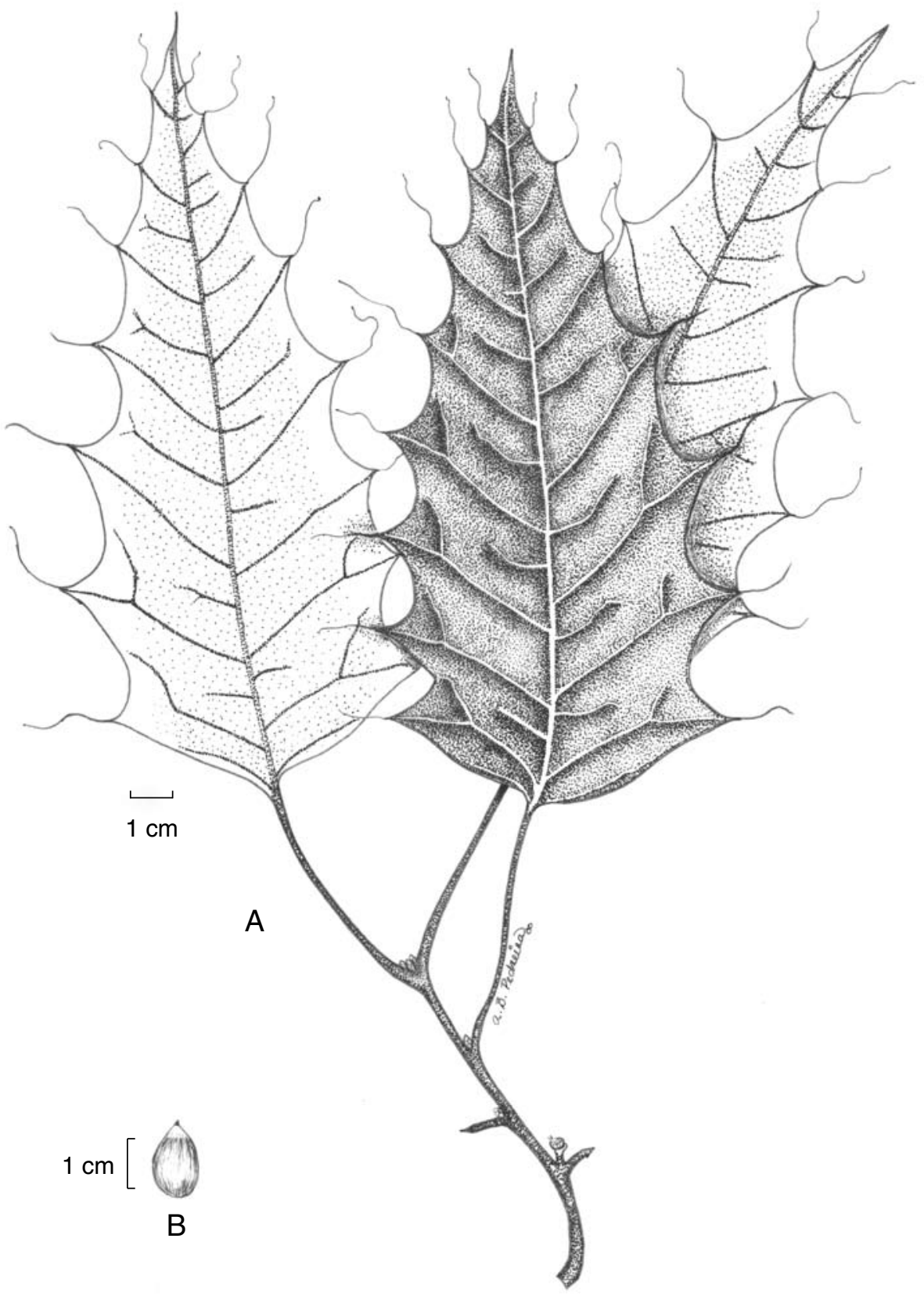

Fig. 2. Quercus albocincta. A. rama con hojas (Breedlove 62881, MEXU); B. fruto (Joyal 2061, MEXU). 
estipitados cortos distribuidos en la lámina, estrellados largos en las axilas de las nervaduras y tricomas glandulares; hojas maduras deciduas, rígidas y coriáceas, elípticas u ovadas, de (4-) 8-17 cm de largo por (3-) 4-8 (-9) cm de ancho, ápice atenuado o largamente atenuado; base cuneada, aguda o redondeada, márgenes engrosados, revolutos, con dientes aristados distribuidos desde la base hasta el ápice, o en las dos terceras partes superiores; dientes 3-6 en cada lado, de más de $20 \mathrm{~mm}$ de largo, aristas de 5-12 mm de largo; nervaduras secundarias 5-11 en cada lado de la lámina de la hoja, pasando directamente hasta los dientes, rectas, con frecuencia curvándose hacia abajo; haz algo lustroso y glabrescente, pronto casi glabro, con tricomas fasciculados estipitados cortos sobre las nervaduras y dispersos, tricomas fasciculados estipitados largos y simples sobre la nervadura primaria, nervaduras impresas; envés casi glabro, amarillento, con tricomas fasciculados estipitados largos en las axilas de las nervaduras, tricomas fasciculados estipitados cortos y glandulares sobre la nervadura primaria, epidermis lisa, nervaduras convexas; pecíolos de 1.5-6 cm de largo por 1-1.5 mm diámetro, glabrescentes; amentos masculinos de 10-15 $\mathrm{cm}$ de largo, con 30 a más de 70 flores, perianto sésil, pubescente, de 2-3 $\mathrm{mm}$ de diámetro, anteras 5 o 6, glabras, elípticas, de 1-1.5 mm de largo, filamentos de 1.5-2 $\mathrm{mm}$ de largo; amentos femeninos de 5-25 mm de largo, con 1-5 flores; fruto anual, solitario o en grupos, en pedúnculos de $0-2 \mathrm{~mm}$ de largo; cúpula hemisférica o a veces turbinada, de 5-12 mm de diámetro, de 4-6 mm de alto, escamas de color castaño, canescentes, ápice truncado o agudo, adpreso; bellotas ovoides, de 6-20 $\mathrm{mm}$ de largo por 5-10 mm de diámetro, incluidas de un tercio a un medio de su largo en la cúpula.

Distribución y ecología. Se le conoce de los estados de Chihuahua, Durango, Sinaloa, Sonora, habitando en bosque de encino y bosque de pinoencino, entre $\operatorname{los} 800$ y 1800 m s.n.m. Florece de febrero a mayo y fructifica de junio a octubre.

Nombres vulgares. Encino-roble, roble.

Ejemplares examinados. MÉXICO. Chihuahua: Batopilas, E de La Bufa, Bye 7761 (MEXU); Creel, La Bufa SE of Creel, Knobloch 418 (ENCB, MEXU); Maguarichi, $34 \mathrm{~km}$ from the Basaseachic-San Juanito, $3.5 \mathrm{~km}$ NE of Maguarichi, Spellenberg 27 (MEXU). Durango: Tepehuanes, Rancho el Purgatorio, González 2529 (MEXU). Sinaloa: Badiraguato, Monte Alto Temeapa, Martínez 113 (MEXU); Badiraguato, $28 \mathrm{~km}$ al E de Badiraguato, Breedlove 62881 (MEXU); 
Choix, Bacayopa, Castro 2226 (MEXU); San Ignacio, San Ignacio, Orozco 4 (ENCB); Surotato, 4 miles W of Surotato along road from Mocosito, Breedlove 15603 (ENCB). Sonora: Santa Ana, 36.8 miles NE of Nuri, 4.2 miles E of Santa Ana, Boutin 3671 (MEXU); Tepocah, 9 roadmiles west of Tepocah, Fischer 6902 (ENCB); Yecora, Rancho de Yerbanis, Joyal 2061 (MEXU); 32 km SE de Bamora, Breedlove 61055, 61029 (MEXU); 11 km E de Santa Rosa, Zimmerman 9900 (MEXU); on road between Tecoripa and Yecora, Spellenberg 9900 (MEXU).

Quercus albocincta se caracteriza por sus hojas elípticas u ovadas, el margen de ellas posee dientes grandes y aristas muy largas. Q. canbyi muestra similitud morfológica con esta especie, pero Q. albocincta se distingue de la primera porque posee mayor número de dientes, aristas y nervaduras y los pecíolos son más largos.

Quercus brenesii Trel., Mem. Nat. Acad. Sci. 20: 186. Lám. 377. 1924. Tipo: Costa Rica, S. Ramón y S. Mateo, Brenes 14520 (holotipo en US!). (Fig. 3).

Árbol de 6-10 m, corteza lisa; ramillas de 1-3 mm de diámetro, rojizas, glabras o casi glabras, con tricomas fasciculados estipitados muy frágiles, cuando están glabras son lustrosas, con lenticelas blancas, protuberantes, hasta de $0.5 \mathrm{~mm}$ de largo; yemas ovoides con el ápice obtuso o agudo, de 1-6 mm de largo por 1-2 mm de ancho, con las escamas pubescentes, principalmente en los márgenes, de color castaño; estípulas lineares, de 6-7 mm de largo por $0.5 \mathrm{~mm}$ de ancho, deciduas antes de la madurez de las hojas, escariosas, con tricomas simples; hojas jóvenes pubescentes, haz con tricomas fasciculados estipitados cortos sobre la nervadura primaria y lámina, y tricomas fasciculados estipitados largos sobre la nervadura primaria, envés con tricomas fasciculados estipitados cortos, estrellados largos y tricomas glandulares vermiformes de color ámbar; hojas maduras subcoriáceas, lustrosas, de color verde-amarillento, angostamente elípticas, de 5-12 cm de largo por 1-3.5 cm de ancho, ápice atenuado, aristado; base cuneada o aguda; márgenes engrosados, cartilaginosos, algo revolutos, con 4-6 dientes de cada lado, divergentes, aristados, distribuidos en la mitad superior de la lámina, nervaduras secundarias 7-11 de cada lado de la lámina de la hoja, las inferiores rectas o ligeramente curvas, las de la mitad superior entran a los dientes y terminan en aristas de 1-4 mm de largo; haz glabro o con tricomas fasciculados estipitados largos sobre la nervadura primaria, más abundantes en la base, con escasos tricomas fasciculados estipitados cortos dispersos sobre la lámina, nervaduras impresas; envés más pálido que el haz, glabro o con escasos tricomas fasciculados estipitados cortos, largos y glandulares vermiformes de color ámbar sobre la nervadura primaria, 
Romero Rangel: Revisión taxonómica del complejo Acutifoliae de Quercus

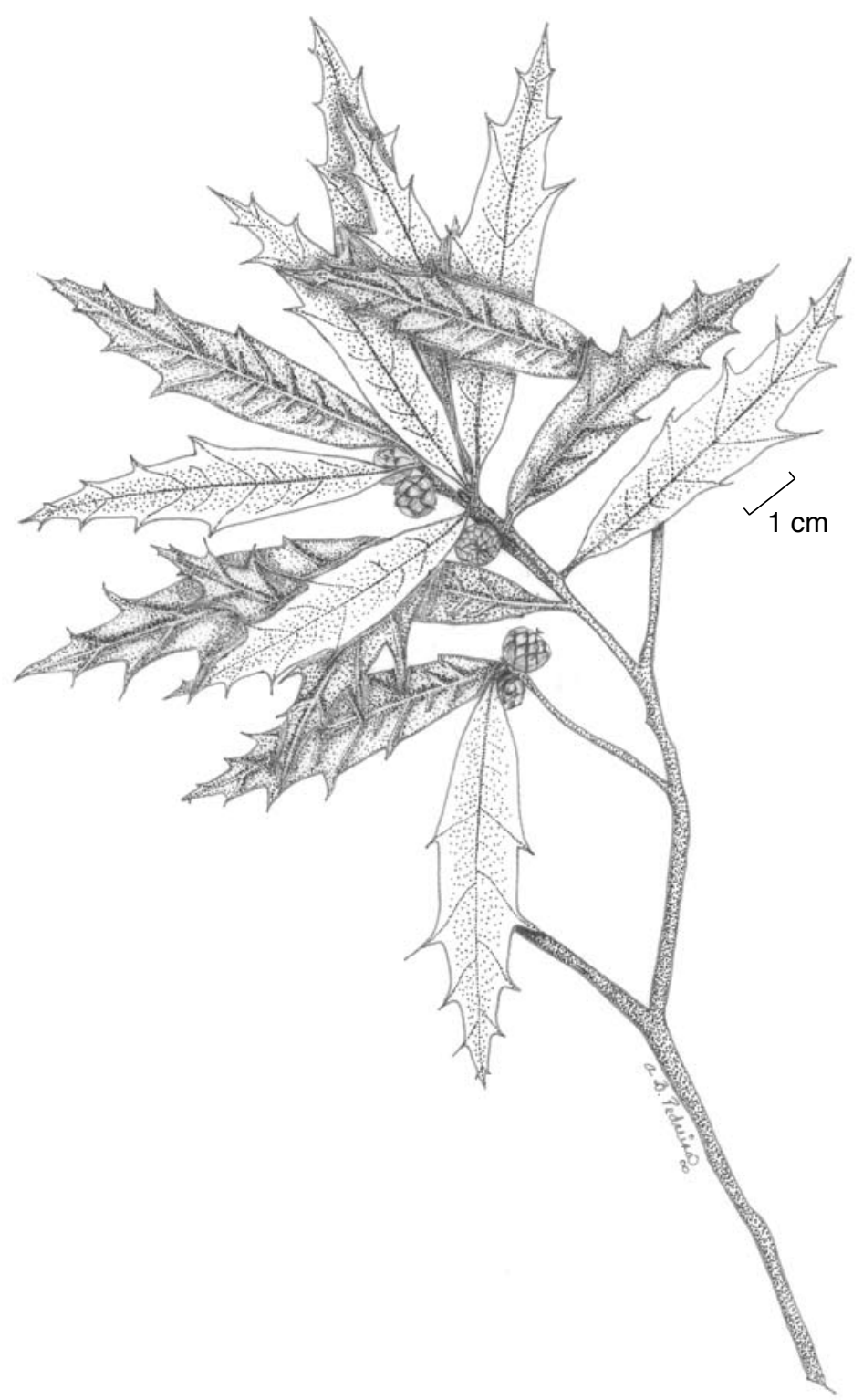

Fig. 3. Quercus brenesii. Ramas fértiles (Sinaca 173, MEXU). 
nervaduras ligeramente convexas, epidermis lisa, blanco papilosa; pecíolos de 3-7 mm de largo por 1-1.5 mm de ancho, glabrescentes, rojizos; amentos masculinos y femeninos desconocidos; fruto anual, solitario o en pares, pedúnculo de 0 a $1 \mathrm{~mm}$ de largo; cúpula hemisférica, de 8-10 mm de diámetro, de 6-8 mm de alto, escamas de color castaño, con ápices obtusos o redondeados, adpresas, pubescentes, con tricomas de color dorado; bellotas ovoides, de 8-10 mm de largo por 6-7 mm de diámetro, incluidas de un tercio a un medio de su largo en la cúpula.

Distribución y ecología. Se le conoce de Veracruz en México, además de Nicaragua y Costa Rica, donde habita en bosque tropical perennifolio y bosque de Quercus, entre los 600 y 1700 m s.n.m. Floración desconocida, fructifica de septiembre a octubre.

Nombres vulgares. Desconocidos.

Ejemplares examinados. COSTA RICA. Puntareanas, Gentry \& Haber 48767 (MO). MÉXICO. Veracruz: Catemaco, Bastonal, $10 \mathrm{~km}$ al NE de Tebanca, camino al poblado de Coyame, Sinaca 174 (MEXU). NICARAGUA. $10 \mathrm{~km}$ al sureste de Tepesomoto, Pueblo Nuevo, Martínez 1763 (ENCB).

Quercus brenesii se caracteriza por sus hojas elípticas, con dientes divergentes, aristados de cada lado, distribuidos en la mitad superior de la lámina, las nervaduras secundarias de la mitad superior con frecuencia dibujan una $S$ al penetrar en los dientes y formar aristas. Esta especie muestra similitud morfológica con Q. cortesii en cuanto a la posición de los dientes en la lámina de la hoja; sin embargo esta última posee mayor número de nervaduras y sus dientes no son divergentes.

Quercus canbyi Trel., Mem. Nat. Acad. Sci. 20: 188. Láms. 379, 380. 1924. Tipo: México, Nuevo León, Monterrey, Pringle 2393 (holotipo en B, isotipo en ENCB!). (Fig. 4).

Quercus alamarensis Müll., J. Arnold Arb. 17: 174. 1936. Tipo: México, Nuevo León, mpio. de Galeana, Alamar on Hacienda Pablillo, Müller 613 (holotipo en A).

Quercus canbyi f. adscendens Trel., Mem. Nat. Acad. Sci. 20: 188. Lám. 380. 1924. Tipo: México, Nuevo León, Pringle 11705 (holotipo en B!).

Quercus canbyi f. attenuata Müll., J. Arnold Arb. 17: 175. 1936. Tipo: México, Nuevo León, mpio. de Villa Santiago, Müller 1347 (holotipo en A). 
Romero Rangel: Revisión taxonómica del complejo Acutifoliae de Quercus

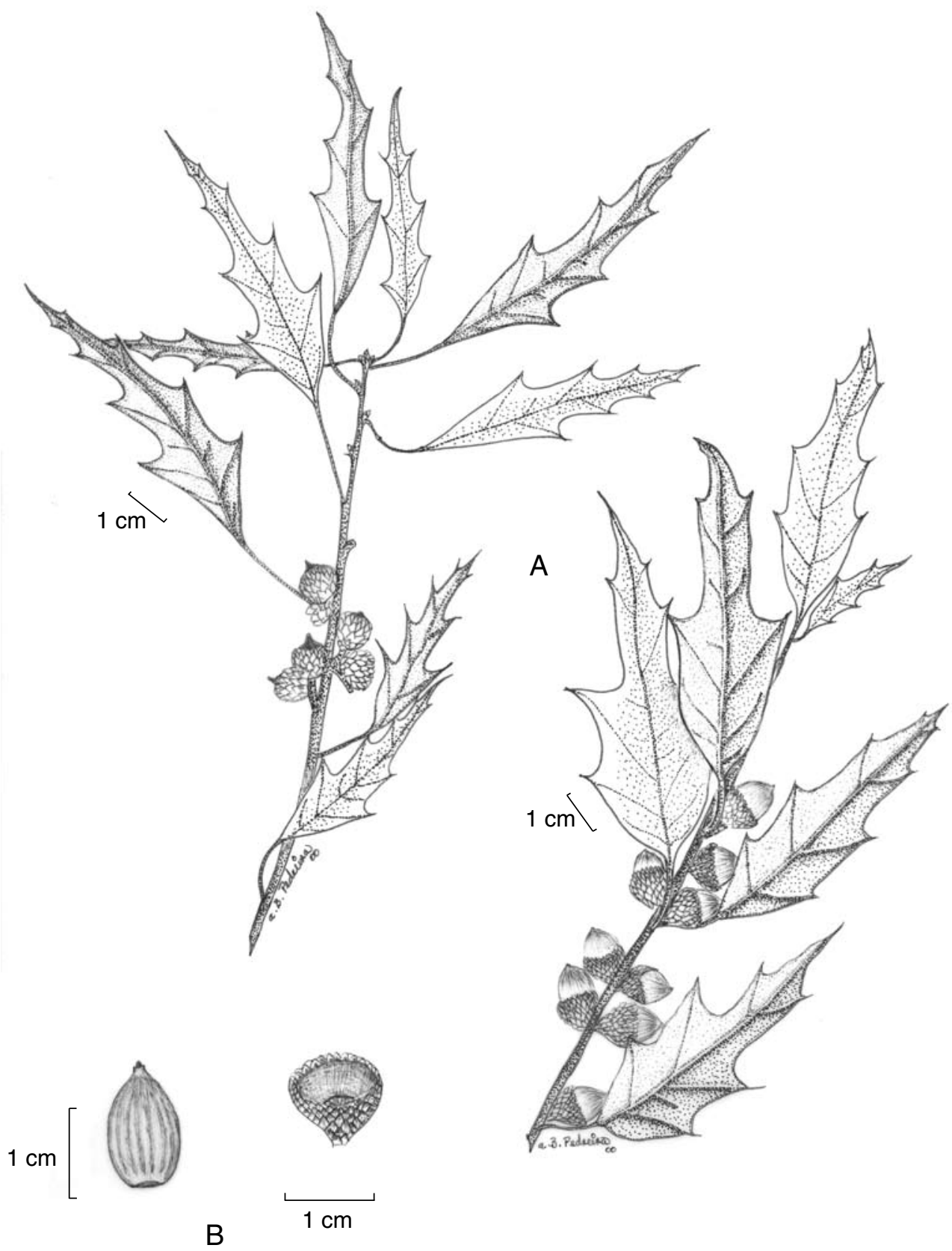

Fig. 4. Quercus canbyi. A. rama fértil; B. fruto y cúpula (González 14166, MEXU). 
Quercus canbyi f. berlandieri Trel., Mem. Nat. Acad. Sci. 20: 188. Lám. 380. 1924. Tipo: México, entre Victoria y Tula, Berlandier 2221 (holotipo en G, isotipo en NY!).

Quercus canbyi f. karwinskii (Trel.) Müll., J. Arnold Arb. 17: 175. 1936. Nombre basado en $Q$. karwinskii Trel.

Quercus canbyi f. pedunculata Müll., J. Arnold Arb. 17: 175. 1936. Tipo: México, Nuevo León, mpio. de Galeana, Puerto de los Pastores, Müller 1009 (holotipo en A).

Quercus canbyi f. setacea Müll., J. Arnold Arb. 17: 175. 1936. Tipo: México, Nuevo León, mpio. de Galeana, Puerto de los Pastores, Müller 1011 (holotipo en A).

Quercus graciliformis Müll., Torreya 34: 120. 1936. Tipo: E.U.A., Texas, Chisos Mts. Müller 565 (holotipo en TEX, isotipo en BH!).

Quercus graciliramis Müll., J. Arnold Arb. 17: 177. 1936. Tipo: México, Nuevo León, Sierra Madre Oriental, Taray to Alamar, about 15 miles southwest of Galeana, Müller 1106 (holotipo en A, isotipo en NY!).

Quercus karwinskii Trel., Mem. Nat. Acad. Sci. 20: 188. Lám. 379. 1924. Tipo: sin datos, Karwinski s. n. (holotipo en M!).

Árbol de 4-15 m de alto, tronco de 20-50 cm de diámetro; corteza oscura y agrietada, ramillas de (0.5-) 1-2.5 mm de diámetro, glabrescentes, rápidamente glabras, entonces obscuras y lustrosas, con lenticelas claras, de 0.5-1 mm de largo o menores; yemas ovoides con el ápice agudo, de (1.5-) 2-6 mm de largo, con las escamas pubescentes, rojizas; estípulas lineares, de (3-) 5-7 mm de largo por 1-1.5 mm de ancho, deciduas muy pronto; hojas jóvenes de color verde oscuro, algo rojizas, haz y envés pubescentes, indumento formado por tricomas fasciculados estipitados y tricomas glandulares; hojas maduras gruesas, rígidas y coriáceas, angostamente lanceoladas a lanceoladas, de 4.5-11 cm de largo por (1-) 2-5 cm de ancho, ápice largamente atenuado, atenuado o agudo, aristado; base cuneada o aguda; márgenes engrosados, revolutos, con dientes de más de $15 \mathrm{~mm}$ de largo, aristados, distribuidos desde la base hasta el ápice, o al menos en las dos terceras partes superiores; los dientes 3-5 en cada lado, aristados, aristas de 2-7 mm de largo; nervaduras secundarias 4-8 en cada lado de la lámina de la hoja, pasando directamente hasta los dientes, rectas o ligeramente curvas; haz verde, glabro o con tricomas fasciculados estipitados cortos y tricomas glandulares dispersos o cerca de las nervaduras; envés casi glabro, amarillento, con tricomas fasciculados estipitados largos en las axilas de las nervaduras y sobre ellas y tricomas fasciculados estipitados cortos y tricomas glandulares de color ámbar o incoloros, dispersos, epidermis lisa; pecíolos de (0.5-) 
1-2.5 cm de largo por 0.5-1 mm de ancho, glabrescentes; amentos masculinos de 3-5 cm de largo, con 20-30 o más flores, perianto sésil, pubescente, de 2-3 $\mathrm{mm}$ de diámetro, anteras 4-6, glabras, elípticas, de 1-2 mm de largo, filamentos de 0.5-1 $\mathrm{mm}$ de largo; amentos femeninos de 5-10 mm de largo, con 1-2 flores; fruto anual o bianual, solitario o en pares, pedúnculo de 0-3 mm de largo; cúpula hemisférica o a veces turbinada, de 7-11 mm de diámetro, de 6-10 $\mathrm{mm}$ de alto, borde delgado, escamas canescentes, con ápices truncados o agudos, adpresas; bellotas ovoides, de 8-17 mm de largo por 7-10 mm de diámetro, de color castaño pálido, incluidas de un tercio a un medio de su largo en la cúpula.

Distribución y ecología. Se le encuentra en la parte suroeste de Estados Unidos de América y México, en los estados de Coahuila, Nuevo León y Tamaulipas, habitando en bosque de encino, bosque de pino-encino, y matorral xerófilo, entre los 600 y 2100 m s.n.m. Florece de marzo a abril y fructifica de julio a noviembre.

Nombres vulgares. Encino blanco, encino colorado, encino de hierba, encino duraznillo.

Ejemplares examinados. MÉXICO. Coahuila: Santa Fé del Pino, Villa Ocampo, Passini 5044, 5119 (ENCB). Nuevo León: Aramberri, 3 km al norte de La Escondida, Marroquín III-25 (MEXU); La Lagunita, González-Medrano 4968; Castellanos, al E de Castellanos, Puig 2956 (ENCB); Chepenque, Mesa de Chepenque, Langman 2891 (MEXU); Galeana, Hinton \& Rzedowski 17286 (MEXU); 3 miles above Iturbide, McVaugh 10559 (MEXU); carretera Galeana-Doctor Arroyo, Robert 308 (ENCB); cerro de los Laureles, Villarreal 2154 (ENCB); cerro Popoi, Villarreal 2152 (MEXU); Middle Santa Bárbara Canyon, Müller 1094 (MEXU); Mesa de la Camisa, Müller 1177 (MEXU); Iturbide, above Iturbide, Hinton 17720 (ENCB); Linares, 57 km oeste of Linares on Hwy 6, Dunn 20127 (ENCB); Montemorelos, cerro de Las Parras, Villarreal 2159 (MEXU); Monterrey, foothills above Monterrey, Pringle 10155 (MEXU); Diente Canyon about 12 miles south of Monterrey, Müller 1305 (MEXU); below Hacienda Vista Hermosa, 2 miles South of Santiago, Manning 53130 (MEXU); Cañón del Diente, zona de la Sierra Madre Oriental, Sánchez 663 (ENCB); cerro de la Silla, Hernández 7210 (ENCB); Potrero Redondo, Rayones 2100 (MEXU); Rayones, Potrero de Las Cabras, Villarreal 2153 (MEXU); Santiago, Villa Hermosa, Martínez 32 (MEXU); 3 km al oeste de V. de Santiago, Valdez (ENCB); Risco Colorado, San Francisco, Garza 2151 (ENCB); Zaragoza, La Cañada, Hinton 24551 (MEXU). Tamaulipas: Ciudad Victoria, al oeste de Ciudad Victoria, Ramírez 
2351 (MEXU); 13 miles southwest of Ciudad Victoria, McVaugh 10499 (MEXU); Cañón de la Presa, Martínez 62, 63 (MEXU); Cañón del Novillo, Castañeda 2754 (MEXU); Ciudad Victoria-Jaumave Hwy over and across mountain range west of Cd. Victoria, Hernández 5123 (MEXU); road Ciudad Victoria toward Jaumave, Sharp 52007 (ENCB); El Picacho, Puig 4762 (MEXU); Jaumave, $12 \mathrm{~km}$ al sur de Ávila y Urbina, González 14166 (MEXU); $30 \mathrm{~km}$ al norte de Jamauve, Kiner s. n. (ENCB); Balcón de Chiue, 20 km al NE de Jaumave, González 12803 (MEXU); San Carlos, La Vegonia, Sierra de San Carlos, Bartlett 10534 (ENCB); Piedra Imán, 2 km al ESE de San José, Briones 1201 (ENCB); San José del Valle, San José del Valle, Puig 2778 (ENCB); Soto la Marina, cerro de los Picachos, Puig 2861, 2881 (ENCB).

Quercus canbyi se caracteriza por sus hojas gruesas, rígidas y coriáceas, angostamente lanceoladas a lanceoladas, márgenes con 3 a 5 dientes grandes y aristados, distribuidos desde la base hasta el ápice, o en las dos terceras partes superiores. Con la finalidad de esclarecer los límites taxonómicos de esta especie, usando un mayor número de caracteres diagnósticos, se hizo un estudio de la arquitectura foliar (Romero R., 2001) a raíz del cual pudo verse que $Q$. canbyi posee la vena primaria muy delgada, las venas secundarias basales formando ángulos más agudos que las demás y venas intersecundarias de tipo compuesto presentes a todo lo largo de la hoja. Los caracteres anteriores se observaron asimismo en los ejemplares de Q. alamarensis Müll., Q. graciliformis Müll. y Q. karwinskii Trel., por lo que se consideraron sinónimos.

Las formas de Q. canbyi propuestas por Trelease (1924) y Müller (1936b) representan ejemplares que difieren en cuanto a las dimensiones de hojas, dientes y frutos; lo que se considera en este trabajo como parte de la variación normal de la especie. También se vio que los caracteres antes mencionados están presentes en dichas formas (Cuadro 1).

Esta especie tiene similitud con Q. albocincta, la cual se distingue de la primera por sus hojas elípticas u ovadas, mayor número de nervaduras, así como dientes, pecíolos y aristas más largos.

Müller (1936b) cita a Quercus canbyi f. subovatifolia Müll. del estado de Nuevo León, México, mencionando que esta forma es un grupo artificial, por lo que ninguno de los ejemplares citados fue designado como tipo.

Quercus conspersa Benth., Pl. Hartw. 91. 1842. Quercus acutifolia var. conspersa (Benth.) A. DC., Prodr. 16(2): 66. 1864. Tipo: Guatemala, Las Casillas, Hartweg 617 (holotipo en CGE, isotipo en NY!). (Fig. 5). 


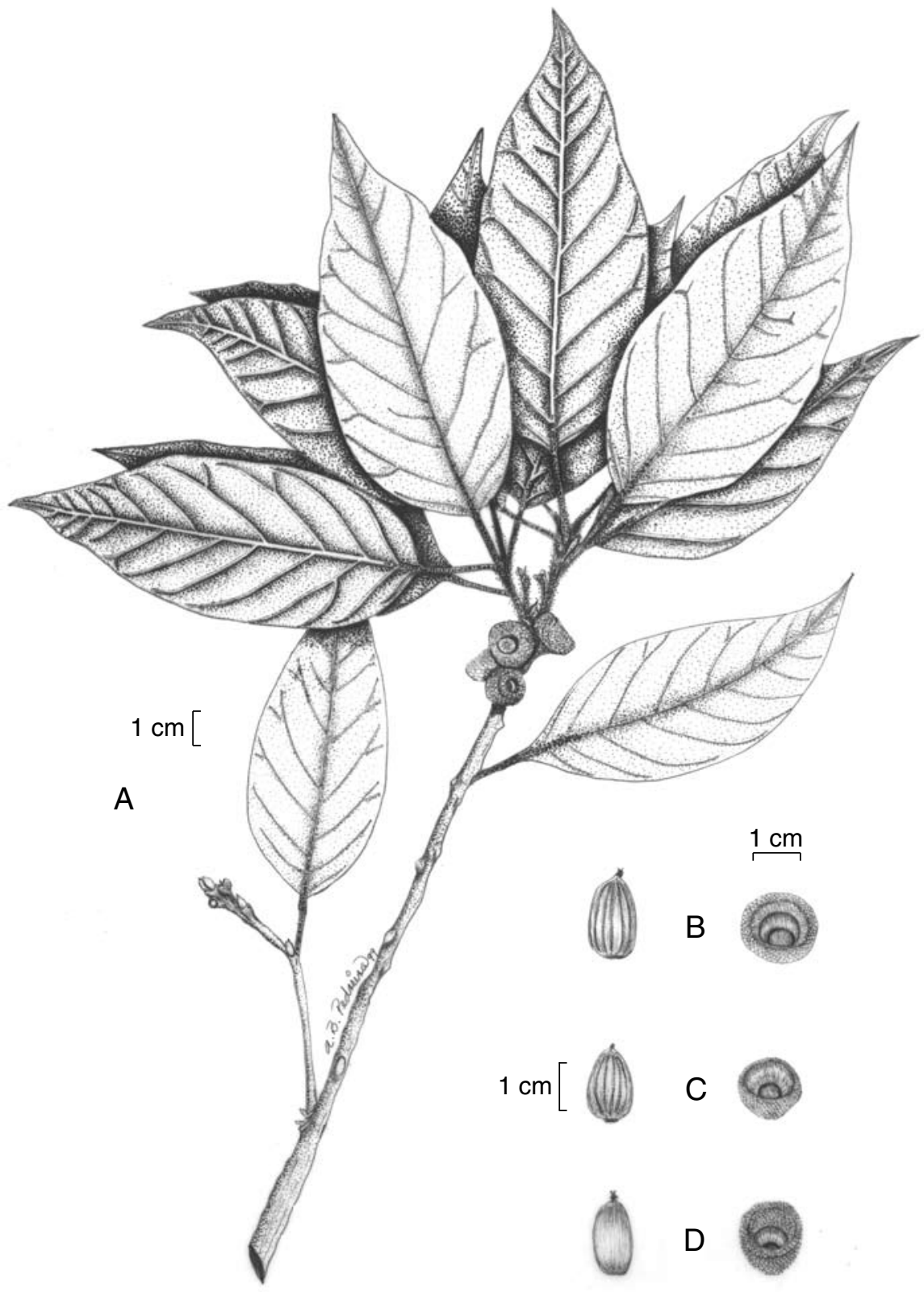

Fig. 5. Quercus conspersa. A. rama fértil con hojas y frutos (Madrigal 3459, ENCB); B-D. variación en frutos y cúpulas (Breedlove 11870, Rzedowski 17366, ENCB; Laughlin 1959, ENCB). 
Quercus correpta Trel., Mem. Nat. Acad. Sci. 20: 153. Lám. 300. 1924. Nombre basado en Q. acutifolia var. microcarpa DC., Prodr. 16(2): 67. 1864. Tipo: Guatemala, sin localidad precisa, Warscewicz 25 (holotipo en G!).

Quercus uruapanensis Trel., Mem. Nat. Acad. Sci. 20: 143. Lám. 276, 277. 1924. Nombre basado en Q. nitida Mart. et Gal., 1843, no Q. nitida Raf. 1838. Tipo: México, Michoacán, Uruapan, Galeotti 121 (holotipo en BR, isotipo en B!).

Árbol de 3-25 m de alto y tronco de 20-50 cm o más de diámetro; corteza oscura y cuadriculada, ramillas de 1-3 (-4) mm de diámetro, glabrescentes, de color rojizo, con numerosas lenticelas claras, hasta de $1 \mathrm{~mm}$ de largo; yemas ovoides con el ápice agudo, de 2-7 mm de largo, con las escamas pubescentes, de color castaño; estípulas lineares, de 3-7 mm de largo por 1-2 mm de ancho, deciduas antes de la madurez de las hojas; hojas jóvenes rojizas, haz y envés pubescentes, con abundantes tricomas fasciculados estipitados cortos, tricomas glandulares y tricomas fasciculados estipitados largos; hojas maduras gruesas, rígidas y coriáceas, deciduas, elípticas, ovadas u obovadas, de 8-18.5 (-21) cm de largo por 2-6 (-9) cm de ancho, ápice acuminado, atenuado o agudo, aristado; base aguda, obtusa o redondeada, márgenes engrosados, revolutos, enteros, nervaduras secundarias 7-14 en cada lado de la lámina de la hoja, ascendentes, rectas o ligeramente curvas, paralelas; haz algo lustroso, de color verde olivo, casi glabro, con tricomas fasciculados estipitados en la base de la nervadura primaria y tricomas glandulares en la base de la nervadura primaria, nervaduras impresas; envés glabrescente, con tricomas glandulares abundantes, a veces escasos, de color ámbar, distribuidos en toda la lámina o cerca de las nervaduras, tricomas fasciculados estipitados cortos dispersos en la lámina y tricomas fasciculados estipitados largos en las axilas de las nervaduras, nervaduras convexas; pecíolos de 6-30 (-37) mm de largo por 0.5-2.0 $\mathrm{mm}$ de ancho, muy engrosados en la base, glabrescentes; amentos masculinos de 4-11 cm de largo, con 20 a más de 50 flores, raquis pubescente y glandular; perianto rojizo o de color púrpura, sésil, pubescente, de 2-2.5 mm de diámetro, anteras 4-6, glabras, oblongas, de 0.8-1.5 mm de largo, filamentos de 1-1.5 mm de largo; amentos femeninos de 6-20 mm de largo, con 1-4 flores; fruto anual o bianual, solitario o en grupos de 2-4, pedúnculo grueso, de 2-20 mm de largo; cúpula hemisférica o a veces turbinada, de 12-18 mm de diámetro, de 6-12 $\mathrm{mm}$ de alto, borde enrollado o a veces recto, escamas canescentes con ápices agudos u obtusos, adpresas; bellota ovoide, de 8-20 mm de largo por 6-18 mm de diámetro, de color castaño pálido, incluida de un tercio a un medio de su largo en la cúpula. 
Distribución y ecología. Se le encuentra en México, en los estados de Jalisco, Michoacán, Guerrero, México, Oaxaca, Chiapas, así como en Guatemala, habitando en bosque de encino, bosque de pino-encino y bosque mesófilo de montaña, entre los 750 y 2440 m s.n.m. Florece de febrero a marzo y fructifica de junio a febrero.

Nombres vulgares. Encino blanco, encino pipitillo, encino rojo, encino teposcohuite.

Ejemplares examinados. GUATEMALA. San Marcos, between San Rafael Pie de la Cuesta and Palo Gordo, Williams 25817 (ENCB). MÉXICO. Chiapas: Ángel Albino Corzo, Jaltenango alrededor de finca Prusia, Breedlove 38534 (ENCB); Cintalapa, 1 km NE de Rizo de Oro, sobre la carretera a Cintalapa, Rzedowski 33087 (MEXU); 5-7 km noroeste of Rizo de Oro road to cerro Baul and Colonia Figueroa, Breedlove 24661 (ENCB); La Trinitaria, Laguna Tzikaw, Monte Bello National Park, Breedlove 29634 (ENCB); Ocosingo, $10 \mathrm{~km}$ southwest of Ocosingo along road to San Cristóbal, Breedlove 27854 (ENCB); Pueblo Nuevo Solistahuacán, 3 km northwest of Pueblo Nuevo Solistahuacán, Thorne 40046 (ENCB); Tenejapa, In the paraje of Kulak'tik, Breedlove 11002 (ENCB); Venustiano Carranza, north side of Aguacatenango near the lake, Raven 20090 (ENCB); north side of Aguacatenango near the lake, Breedlove 20091 (ENCB); Villa Corzo, the east base of cerro de Tres Picos near cerro Bola along a logging road southwest of Colonia Agrónomos Mexicanos, Breedlove 23960 (ENCB). Guerrero: Xaltianguis, between Cajelito and Piedrimang on cerro W of Xaltianguis, ca. $50 \mathrm{~km} \mathrm{~N}$ of Acapulco, Müller 9199 (ENCB); below Cajetito on cerro W of Xaltianguis, ca. 50 km N of Acapulco, Müller 9195 (MEXU); Jalisco: Autlán, $1.5 \mathrm{~km}$ al suroeste del puerto de los Mazos, al oeste de Autlán por la carretera a Barra de Navidad, Villarreal de Puga 1895 (ENCB); $10 \mathrm{~km}$ al norte de Autlán, Las Mesas de San Francisco, 3 km al oeste de la Mina San Francisco, Villarreal de Puga 2052 (ENCB); SE of Puerto Los Mazos on road from Autlán to Barra de Navidad, Breedlove 61719 (MEXU); El Guisar, SE an above El Chante going towards El Guisar, Breedlove 61747 (MEXU); Jilotlán de los Dolores, km 82 brecha Tecatitlán-Jilotlán de los Dolores, Santana 1071 (ENCB); Tamazula de Gordiano, El Montozo, Villarreal de Puga 1623 (ENCB); cerca de Agua Hedionda, 45 km al E de El Aserradero, Rzedowski 1072 (ENCB). México: Tejupilco, Nanchititla, al oeste de Luvianos, Rzedowski 20701(ENCB); Temascaltepec, $5 \mathrm{~km}$ al suroestedeTemascaltepec sobre carretera a Tejupilco, Rzedowski 20807 (ENCB). Michoacán: Ario de Rosales, $5 \mathrm{~km}$ S of Ario de Rosales, Müller 9240, 9241 (MEXU); 9 km al suroeste de Ario de Rosales hacia La Huacana, Torres 347 (ENCB, MEXU); cerro de Tipitarillo, 12 km al 
noroeste de Ario de Rosales, Martínez 2800 (ENCB); Ibid., Martínez 2801 (ENCB, MEXU); Tacámbaro, cerro del Coco, Viveros 197 (ENCB); Tumbiscatío, Arroyo de Juan Cruz, Aguilar 132 (ENCB); Uruapan, cerro de La Charanda Villarreal de Puga 1681 (MEXU); Barranca below the Cascada de Tzararacua near Uruapan, Pringle 8840 (ENCB); alrededores de Uruapan, Rojas \& Romero 4741 (IZTA); Campo experimental forestal en la Barranca de Cupatitzio, Zamora 4832 (MEXU); Campo experimental forestal del INIF en la Barranca de Cupatitzio, González 745 (MEXU); cerro La Charanda, al norte de Uruapan, González 1681 (MEXU); cerro Los Puercos, población próxima las Tejerías, s. colector sitio 114-11-21 (ENCB); La Tzararacua, Díaz-Luna 3210 (MEXU); La Tzararacua, Madrigal 3331 (ENCB); Ziracuaretiro, 5 km NE de San Andrés Corú, Díaz-Luna 3605 (ENCB), 3695 (MEXU); Zitácuaro, Zitácuaro-Nandio, Hinton 13195 (ENCB). Oaxaca: Quiatoni, $3 \mathrm{~km}$ al noroeste de Unión Juárez camino Río Minas-Peña Colorada, Acosta 1550 (ENCB); San Juan La Jarcia, km 99 Tehuantepec a Oaxaca, Chavelas 4832 (MEXU); Santo Domingo Tehuantepec, cerro El Arenal, al norte de Buenos Aires, Campos 3972 (MEXU).

Q. conspersa se caracteriza por sus hojas gruesas, rígidas y coriáceas, elípticas, ovadas u obovadas, ápice aristado, márgenes engrosados, revolutos y enteros, y llevando tricomas glandulares escasos o abundantes de color ámbar; con frecuencia los márgenes de la cúpula están enrollados. Q. uruapanensis Trel. posee hojas elípticas y los caracteres diagnósticos antes citados, por lo que se consideró sinónimo de $Q$. conspersa. Además, la descripción de la arquitectura foliar mencionada en el estudio de Romero R. (2001), siguiendo lo propuesto por Hichey (1973), permitió ver que ambos taxa poseen los siguientes caracteres: vena primaria de tamaño moderadamente grueso, venas secundarias con ángulos de divergencia uniforme, venas intersecundarias distribuidas en la parte media de la hoja, areólas de tamaño mediano, cuadrangulares y disposición orientada; lo que confirmó, junto con los caracteres diagnósticos mencionados al principio, la condición de sinonimia. No se encontraron caracteres que permitieran definir a $Q$. uruapanensis como especie distinta a $Q$. conspersa.

Müller (1942a) consideró como sinónimo de esta especie a Quercus correpta Trel. (Cuadro 1).

Quercus cortesii Liebm., Overs. Dansk Vidensk. Selsk. Forhandl. p.175. 1854. Tipo: México, Veracruz, Hacienda de Jovo a Huitamalco, Liebmann 3462, 3464 (sintipos en C!). (Fig. 6). 
Romero Rangel: Revisión taxonómica del complejo Acutifoliae de Quercus

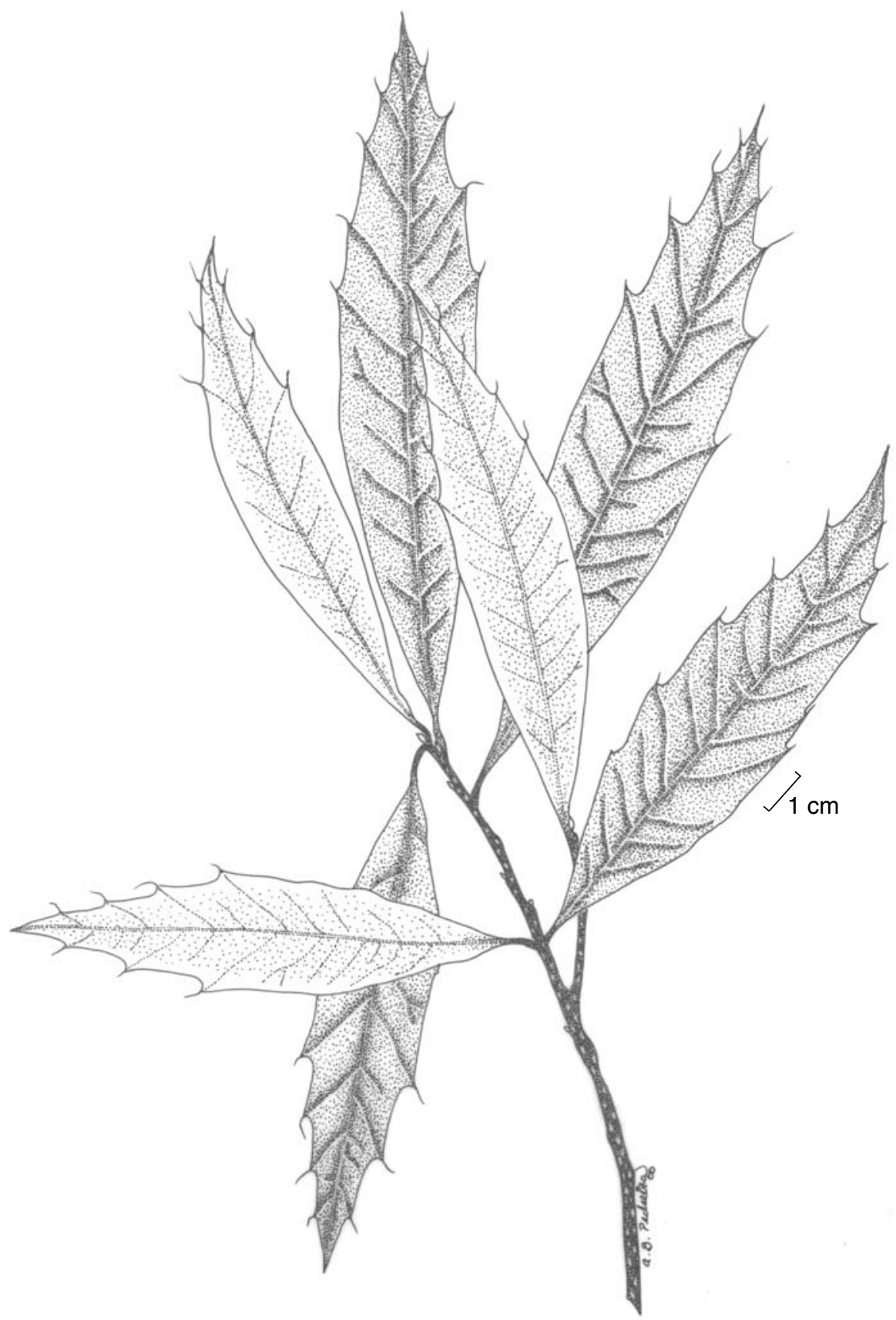

Fig. 6. Quercus cortesii. Rama con hojas (Liebmann 3463, C). 
Árbol con la corteza oscura y agrietada, ramillas de 0.5-1.5 mm de diámetro, glabras o casi glabras, rojizas, obscuras, lustrosas, con lenticelas claras, de 0.5-1 mm de largo; yemas ovoides con el ápice obtuso, de 1-3 mm de largo, con las escamas glabras, a veces con escasos tricomas, de color castaño; estípulas deciduas antes de la madurez de las hojas; hojas jóvenes casi glabras, haz con escasos tricomas fasciculados estipitados cortos sobre la lámina, envés con escasos tricomas fasciculados estipitados cortos distribuidos en la lámina, y mechones de tricomas fasciculados estipitados largos en las axilas de las nervaduras; hojas maduras delgadas, angostamente elípticas o angostamente lanceoladas, de 7-16 cm de largo por 1.5-3.5 cm de ancho, ápice atenuado, a veces agudo, aristado; base atenuada, a veces aguda; márgenes engrosados, ligeramente revolutos, con 4-6 dientes de cada lado, aristados, no divergentes, distribuidos en la mitad superior de la lámina, aristas de 2-6 mm de largo, nervaduras secundarias 10-15 en cada lado de la lámina de la hoja, ascendentes, por lo general rectas y paralelas, a veces algo sinuosas, las superiores pasan directamente a los dientes, terminando en una arista; haz lustroso, glabro, a veces con pocos tricomas fasciculados estipitados cortos en la base de la nervadura primaria; envés más pálido que el haz, nervaduras convexas, casi glabro, con escasos tricomas fasciculados estipitados cortos cerca de la nervadura primaria, tricomas fasciculados estipitados largos en las axilas de la nervaduras, epidermis lisa y papilosa; pecíolos delgados, de 5-10 $\mathrm{mm}$ de largo por $1 \mathrm{~mm}$ de ancho, glabros o con algunos tricomas fasciculados estipitados en la base; amentos masculinos y femeninos desconocidos; fruto anual, solitario o en pares, cúpula hemisférica o a veces turbinada, de 9-20 mm de diámetro, de 7-15 mm de alto, borde recto, a veces enrollado, escamas de color castaño, pubescentes, ápices obtusos, adpresos; bellotas ovoides, de 11-17 mm de largo por 9-14 mm de diámetro, de color castaño pálido, incluidas de un tercio a un medio de su largo en las cúpulas.

Distribución y ecología. Se le encuentra en México en los estados de Chiapas y Puebla; además, en Belice, Costa Rica, Honduras, Nicaragua y Panamá, habitando en bosque de encino. Florece en abril y fructifica de septiembre a noviembre.

Nombres vulgares. Desconocidos.

Ejemplares examinados. BELICE. Toledo: Columbia Forest Reserve, Holst 4307 (MO). COSTA RICA. Puntareanas, Haber y Bello 7274 (MO). HONDURAS. Comayagua, Yunker, Dawson y Mouse 6204 (MO). MÉXICO. Chiapas: Jitotol, Breedlove y Thorne 21366 (MO). Veracruz: Hacienda de Jovo to Huitamalco, 
Liebmann 27, 28, 29 (ST), Liebmann 3462 (C, ST), Liebmann 3463(C), Liebmann 3464 (C, ST). NICARAGUA. Matagalpa, sin localidad, Moreno 1044 (MO). PANAMÁ. Chiriquí, Folsom, Dressler y Dressler 5374 (MO).

Quercus cortesii se caracteriza por sus hojas delgadas, angostamente elípticas o angostamente lanceoladas, con 4-6 pares de dientes aristados distribuidos en la mitad superior de la lámina, nervaduras secundarias por lo general rectas y paralelas. Esta especie muestra similitud con Q. brenesii en cuanto a la posición de los dientes en la lámina de la hoja, sin embargo la última se puede reconocer por sus hojas con menor número de nervaduras secundarias y porque sus dientes son divergentes.

Quercus furfuracea Liebm., Overs. Dansk Vidensk. Selsk. Forhandl. 1854: 189. 1854. Tipo: México, Puebla, Chinautla, Liebmann 3438 (holotipo en C!). (Fig. 7).

Árbol o arbusto de 3-6 m de alto; ramillas de 1-3 mm de diámetro, densamente pubescentes, con el indumento amarillento, que perdura por varias estaciones, formado por tricomas fasciculados estipitados largos y tricomas fasciculados estipitados cortos, superficie de epidermis rojiza o de color castaño oscuro; lenticelas casi inconspicuas, de menos de $0.5 \mathrm{~mm}$ de largo; yemas ovoides, rojizas, lustrosas, con el ápice obtuso a redondeado, de 1-3.6 mm de largo, con las escamas pubescentes; estípulas lineares, de 4-7 mm de largo por 0.5-1 mm de ancho, deciduas antes de la madurez de las hojas; hojas jóvenes densamente pubescentes, haz con tricomas fasciculados estipitados largos sobre la nervadura primaria y tricomas fasciculados estipitados cortos dispersos sobre la lámina, envés con tricomas fasciculados estipitados largos sobre la nervadura primaria y en las axilas de las nervaduras secundarias y tricomas fasciculados estipitados dispersos en la lámina; hojas maduras gruesas, rígidas y coriáceas, deciduas, elípticas u ovadas, de 4.5-10 cm de largo por 2-5.5 cm de ancho, ápice atenuado o agudo, aristado; base obtusa, redondeada o cordada; márgenes engrosados, revolutos, con dientes aristados, a veces muy cortos, distribuidos desde la base hasta el ápice o en las dos terceras partes superiores; los dientes aristados 3-10 en cada lado, ascendentes, aristas de 1-6 mm de largo; nervaduras secundarias 4-8 en cada lado de la lámina de la hoja, pasando directamente hasta los dientes, ascendentes, rectas a ligeramente curvas, paralelas; haz algo lustroso, de color verde olivo, pubescente, con tricomas fasciculados estipitados largos sobre la nervadura primaria y tricomas fasciculados estipitados cortos dispersos en la lámina, nervaduras planas o impresas; envés densamente pubescente, con el indumento persistente, canescente, formado por 


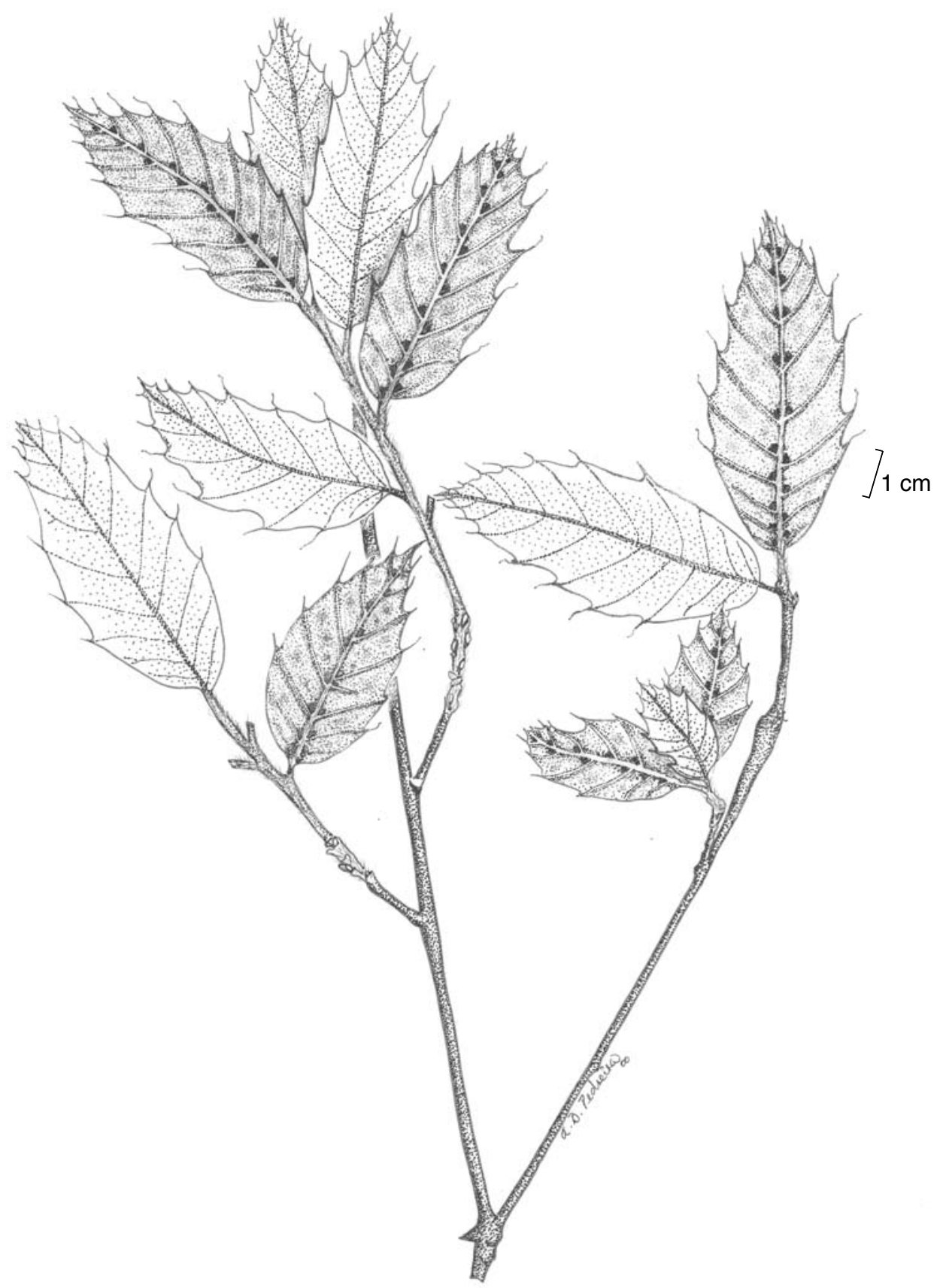

Fig. 7. Quercus furfuracea. Rama con hojas (Rzedowski 6016, ENCB). 
tricomas fasciculados estipitados largos en las axilas de las nervaduras secundarias y tricomas fasciculados estipitados cortos dispersos en la lámina; epidermis lisa y papilosa; nervaduras convexas, la primaria rojiza, las secundarias amarillentas, pálidas; pecíolos de 1-3 cm de largo por 0.5-1 mm de diámetro, muy pubescentes, glabrescentes, indumento amarillento; amentos masculinos desconocidos; amentos femeninos de 10-20 mm de largo, con una a varias flores; fruto bianual o anual, solitario o en pares, pedúnculo de 10-20 mm de diámetro; cúpula hemisférica o a veces turbinada, de 10-12 $\mathrm{mm}$ de diámetro, de 6-7 $\mathrm{mm}$ de alto, escamas de color rojizo, pubescentes, ápices obtusos a redondeados, adpresas; bellotas ovoides, de 8-11 mm de largo por 7-10 mm de diámetro, incluidas un medio de su largo en las cúpulas.

Distribución y ecología. Se le conoce de México, de los estados de San Luis Potosí y Puebla, habita en bosque de encino, entre los 1300 y 2000 m s.n.m. Floración desconocida, fructifica en septiembre.

Nombres vulgares. Encino blanco, encino de asta, encino laurelillo, encino rojo, encino saucillo, encino sencillo, encino teposcohuite.

Ejemplares examinados. MÉXICO. Puebla: Chignahuapan, Venta Ahumada, Romero y Rojas 4830 (IZTA). San Luis Potosí: Guadalcázar, 7 km al sureste de Guadalcázar, Rzedowski 6016 (ENCB); 15 km al norte de Guadalcázar, sobre el camino a Murciélago, Rzedowski 6023 (ENCB); 8 km al noroeste de Guadalcázar, Rzedowski 5974, 5983 (ENCB, MEXU); 8 km al sur de Guadalcázar, Rzedowski 5953 (ENCB, MEXU); Zaragoza, km 53 carretera San Luis Potosí-Ríoverde, entre Las Rusias y Cruz de Encino, Takaki 2029 (ENCB).

Quercus furfuracea se caracteriza por sus ramillas y hojas densamente pubescentes por una o más temporadas; las hojas son gruesas, rígidas y coriáceas, elípticas u ovadas, de tamaño mediano (4.5-10 cm de largo por 2-5.5 cm de ancho).

Quercus skinneri Benth., Gard. Chron. 1: 16. 1841. Tipo: Guatemala, In montium declivitate versus Oceanum Pacificum, Acatenango, Medio Monte et Quezaltenango, Hartweg 615 (holotipo en K, isotipo en NY!). (Fig. 8).

Quercus chiapasensis Trel., Proc. Amer. Philos. Soc. 54: 9. Lám. 2. 1915. Tipo: México, Chiapas, Finca Irlanda, Purpus 6999 (holotipo en ILL, isotipo en NY!). 


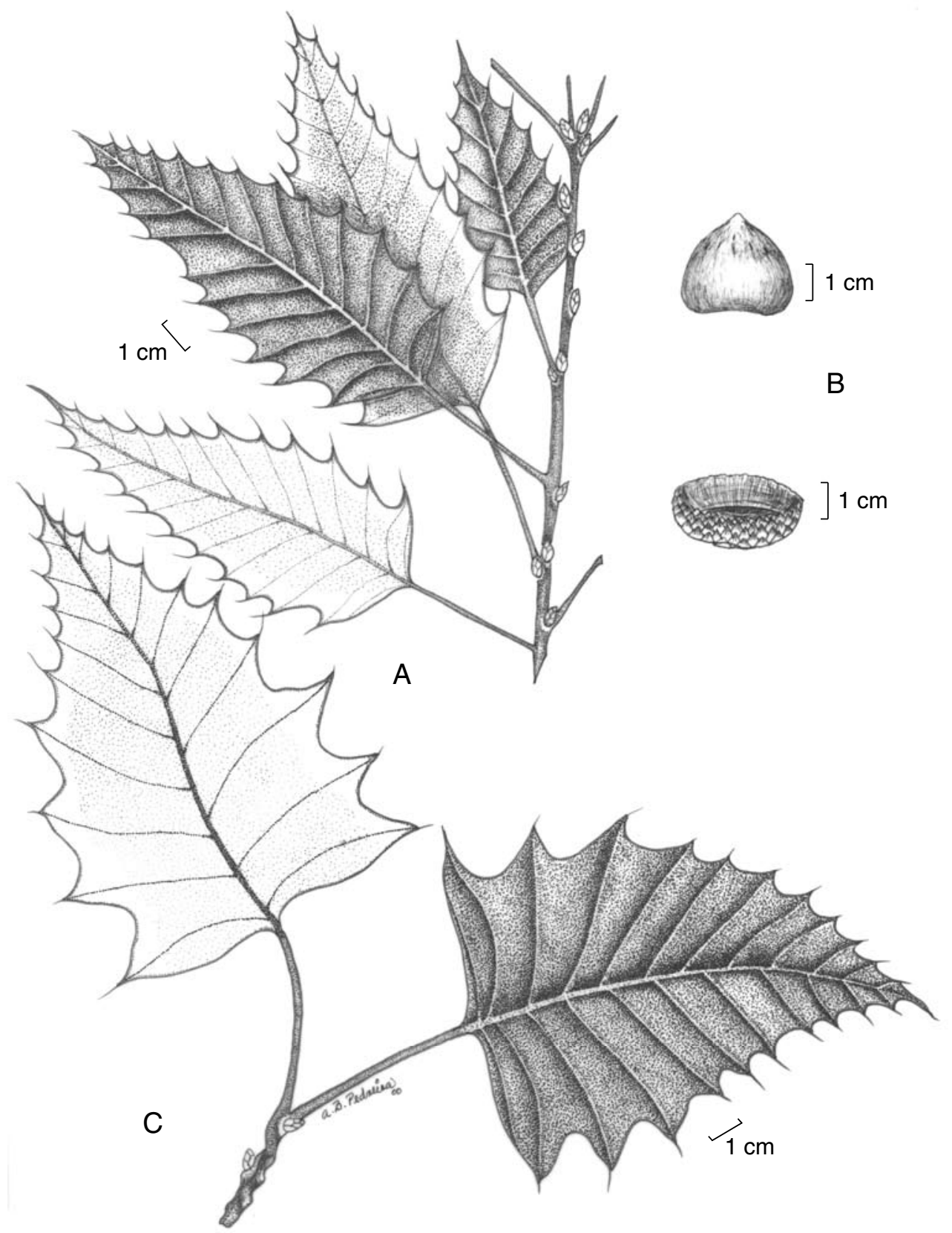

Fig. 8. Quercus skinneri. A. rama con hojas; B. fruto y cúpula (Cházaro 774, MEXU); C. rama con hojas (Beaman 5437, MEXU). 
Quercus chiapasensis f. cuneifolia Trel., Proc. Amer. Philos. Soc. 54: 9. Lám. 2. 1915. Tipo: México, Chiapas, Finca San Juan de las Chicharras, Reeves 3 (holotipo en ILL).

Quercus chiapasensis f. falcilobata Trel., Mem. Nat. Acad. Sci. 20: 186. Lám. 375. 1924. Tipo: México, Chiapas, Finca San Juan de las Chicharras, Reeves 5 (holotipo en ILL!).

Quercus chiapasensis f. flagellata Trel., Mem. Nat. Acad. Sci. 20: 186. 1924. Tipo: México, Chiapas, Finca San Juan de las Chicharras, Reeves 6 (holotipo en ILL!).

Quercus chiapasensis f. longipes Trel., Mem. Nat. Acad. Sci. 20: 186. 1924. Tipo:

México, Chiapas, Finca San Juan de las Chicharras, Reeves 1 (holotipo en ILL!).

Quercus chiapasensis f. petiolata Trel., Mem. Nat. Acad. Sci. 20: 186. 1924. Tipo:

México, Chiapas, Finca San Juan de las Chicharras, Reeves 8 (holotipo en ILL!).

Quercus chiapasensis f. subcuneata Trel., Mem. Nat. Acad. Sci. 20: 186. 1924. Tipo: México, Chiapas, Finca San Juan de las Chicharras, Reeves 9 (holotipo en ILL!).

Quercus grandis Liebm., Over. Dansk Vidensk. Selsk. Forhandl. 1854: 183. Lám. 372-373. 1854. Tipo: Guatemala, sin localidad precisa, Warscewicz 20 (holotipo en B!).

Quercus grandis var. tenuipes Trel., Mem. Nat. Acad. Sci. 20. 185. Lám. 373. 1924. Tipo: México, Oaxaca, Lalona, Chinantla, Galeotti 135 (holotipo en G).

Quercus hemipteroides Müll., Amer. Midl. Nat. 18: 853. 1937. Tipo: Guatemala, Quiché, Finca San Francisco, Skutch 1875 (holotipo en ILL!).

Quercus salvadorensis Trel., in Standley, Field Mus. Bot. Ser. 8: 5. 1930. Tipo: El Salvador, Cuscatlán, summit of Cerro del Guayabal, Calderón 1981 (holotipo en US).

Quercus trichodonta Trel., Field Mus. Bot. Ser. 17: 358. 1938. Tipo: Honduras, Comayagua, El Achote, above Siguatepeque, Yuncker, Dawson y Youse 6116 (holotipo en ILL!, isotipo en MO).

Árbol de (7-) 15-40 m de alto; corteza oscura y agrietada, ramillas de 1$4 \mathrm{~mm}$ de diámetro, glabras, rojizas obscuras o a veces más claras, lustrosas, con lenticelas claras, por lo general menores de $1 \mathrm{~mm}$, a veces hasta de $1.5 \mathrm{~mm}$ de largo; yemas ovoides con el ápice obtuso, de 4-7 mm de largo, con las escamas glabras, a veces con escasos tricomas en sus ápices, de color castaño; estípulas 
lineares, pubescentes, de 7-15 mm de largo por 1-2 mm de ancho, deciduas antes de la madurez de las hojas; hojas jóvenes densamente pubescentes, el haz y envés están cubiertos por tricomas fasciculados estipitados, simples y glandulares que no dejan ver la epidermis, las aristas se observan a veces algo enrolladas en el margen de la lámina de la hoja; hojas maduras delgadas, a veces semicoriáceas, elípticas u ovadas, de (6-) 7.5-22 (-30) cm de largo por (2.5) 3-9 (-12) cm de ancho, ápice atenuado, acuminado o agudo, aristado; base truncada, cordada u obtusa, a veces en hojas jóvenes se observa decurrente; márgenes engrosados, planos o ligeramente revolutos, con 10-13 dientes aristados distribuidos de cada lado en todo el margen de la lámina, aristas de 5-10 mm de largo, nervaduras secundarias 9-15 en cada lado de la lámina de la hoja, ascendentes, rectas y paralelas, pasando directamente hasta los dientes y terminando en una arista; haz opaco o algo lustroso, glabro, a veces con pocos tricomas fasciculados estipitados cortos y tricomas glandulares sobre o cerca de la nervadura primaria; envés más pálido que el haz, glabro o con escasos tricomas fasciculados estipitados cortos y tricomas glandulares cerca de la nervadura primaria y/o tricomas fasciculados estipitados largos en las axilas de las nervaduras, epidermis lisa y papilosa; pecíolos delgados, de 2.5-7 cm de largo por $1.5 \mathrm{~mm}$ de ancho, glabros o con algunos tricomas fasciculados estipitados cortos; amentos masculinos de $7 \mathrm{~cm}$ de largo, con pocas flores o a veces hasta más de 40, perianto sésil, muy pubescente, de $2-3 \mathrm{~mm}$ de diámetro, anteras 4-6, glabras, elipsoidales, filamentos de $1-2 \mathrm{~mm}$ de largo, amentos femeninos de $5 \mathrm{~mm}$ de largo, con 1 a 2 flores; fruto bianual, solitario o en pares, pedúnculo de 5-15 mm de longitud; cúpula hemisférica, de 20-45 mm de diámetro, de 8-20 mm de alto, escamas de color castaño, pubescentes, ápices obtusos, adpresos; bellotas ovoides, de (15) 20-50 mm de largo por 20-50 mm de diámetro, de color castaño pálido, incluidas un cuarto o menos de su largo en las cúpulas.

Distribución y ecología. Se le encuentra en México en los estados de Chiapas, Oaxaca, Tamaulipas y Veracruz, también en Guatemala, El Salvador y Honduras, habita en bosque de encino, bosque tropical caducifolio y perennifolio, bosque mesófilo de montaña y bosque de encino-pino, entre los 700 y 2200 m s.n.m. Florece de febrero a marzo y fructifica de julio a diciembre.

Nombres vulgares. Encino, roble blanco.

Ejemplares examinados. EL SALVADOR. Sonsonete, Reyna de Aguilar 1481 (MO). GUATEMALA. Solota, Kellerman 6061 (MO). HONDURAS. Francisco 
Morazán, Molina 3156 (MO). MÉXICO. Chiapas: El Triunfo, west falda of Sierra Madre de Chiapas, Matuda 18047 (ENCB). Oaxaca: Buena Vista, Buena Vista, MacDougall 2440 (MEXU); Guevea de Humboldt, cerro de Picacho, 3 km al E de la Cumbre, Campos 3724 (MEXU); La Gloria, La Gloria, MacDougall 2439 (ENCB). Tamaulipas: Gómez Farías, Rancho del Cielo a 11 km al norte de Gómez Farías, González 7407 (MEXU). Veracruz: Santa Martha, Santa Martha, Sousa 3589 (MEXU); Soteapan, entre San Fernando y Santa Marta, San Pedro, Soteapan, Cházaro 774 (MEXU); Sierra de Santa Marta al NE de Ocotal Grande, Beaman 5437 (MEXU).

Quercus skinneri se caracteriza por sus hojas maduras delgadas, a veces semicoriáceas, elípticas u ovadas, hasta de $30 \mathrm{~cm}$ de largo y $12 \mathrm{~cm}$ de ancho, base amplia (truncada, cordada u obtusa); pecíolos largos y delgados (de 2-7 cm de largo por $1.5 \mathrm{~mm}$ de ancho) y frutos hasta de $50 \mathrm{~mm}$ de largo por $50 \mathrm{~mm}$ de diámetro. Müller (1942a) indica que Q. skinneri es una de las especies más polimórficas de América Central y que las características que pueden llevar a conclusiones erróneas son la longitud del pecíolo y la forma y el tamaño del fruto. Los sinónimos señalados fueron determinados por Müller (1942a) y después de haber examinado todo el material, la autora concuerda con tal decisión.

Quercus uxoris McVaugh, Contr. Univ. Mich. Herb. 9: 513. 1972. Tipo: México, Jalisco, Talpa de Allende, 5-8 miles above La Cuesta, below the pass to Talpa de Allende, McVaugh 20292 (holotipo en MICH!, isotipo en ENCB). (Fig. 9).

Árbol de (7-) 15-35 (-45) m de alto y tronco de hasta 1 m o más de diámetro; ramillas de 2-5 mm de diámetro, densamente pubescentes con indumento de color dorado o castaño amarillento, formado por tricomas fasciculados estipitados largos y tricomas glandulares, que persiste una a varias temporadas, con el tiempo se ennegrece, corteza de color castaño-rojizo, con lenticelas claras, hasta de 1 mm de largo; yemas ovoides con el ápice agudo a obtuso, de 2-7 mm de largo, por 1.5-4 mm de ancho, con las escamas pubescentes, de color castaño; estípulas lineares, escariosas, membranosas, de (7-) 8-12 (-15) mm de largo, deciduas antes de la madurez de las hojas, las de las yemas terminales persisten por más tiempo; hojas jóvenes muy pubescentes, con abundantes tricomas glandulares y tricomas fasciculados estipitados cortos y largos, indumento de color dorado; hojas maduras rígidas y semicoriáceas o coriáceas, elípticas u ovadas, de (8-) 9-25 (-30) cm de largo por (3-) 6-11 cm de ancho, ápice atenuado o acuminado, aristado; base aguda, obtusa 


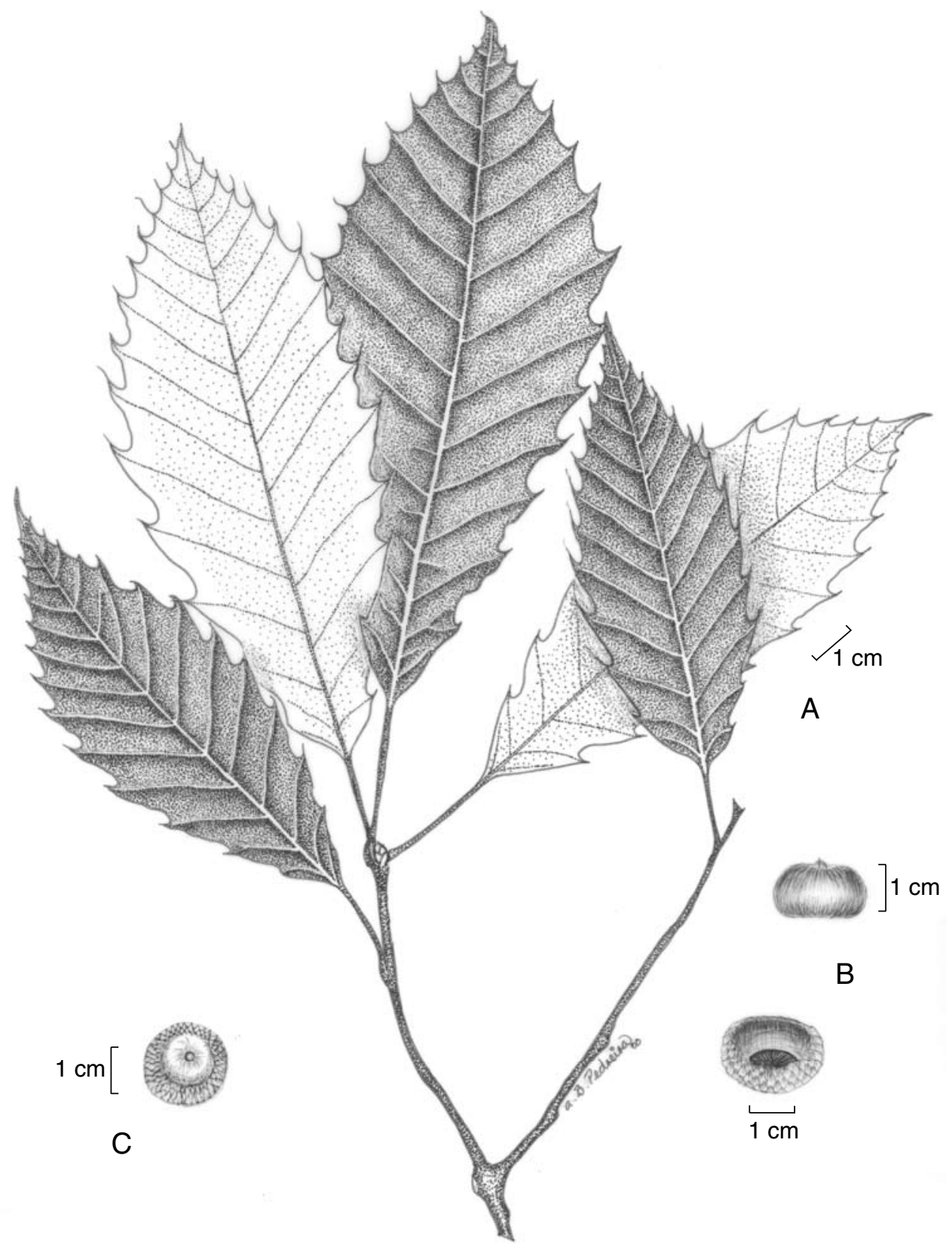

Fig. 9. Quercus uxoris. A. rama con hojas; B. fruto y cúpula; C. fruto joven (Madrigal 2488, ENCB). 
o redondeada; márgenes engrosados, revolutos, con dientes aristados distribuidos desde la base hasta el ápice, o en las dos terceras partes superiores; dientes 9-15 (-18) en cada lado, largos o muy cortos, ascendentes, aristas de 2-8 mm de largo; nervaduras secundarias (7-) 9-14 en cada lado de la lámina de la hoja, pasando directamente hasta los dientes; haz verde, glabrescente, glabro en la madurez, con tricomas glandulares y tricomas fasciculados estipitados largos sobre la nervadura primaria, nervaduras impresas, la primaria algo elevada en la base; envés amarillento, glabrescente, con tricomas glandulares abundantes cerca de la nervadura primaria, tricomas fasciculados estipitados largos en las axilas de las nervaduras secundarias y tricomas fasciculados estipitados cortos sobre la lámina y nervaduras, liso a papiloso, nervaduras impresas; pecíolos pubescentes, glabrescentes, de 12-40 (-50) mm de largo por 0.5-2 (-3) mm de ancho; amentos masculinos de 6-13 cm de largo, llegando a contener más de 70 flores, perianto sésil, muy pubescente, de 1.5-2.5 mm de diámetro, anteras 6, apicuadas, glabras, elípticas, de 1-3 mm de largo, filamentos de 1-2 mm de largo; amentos femeninos de 5-10 mm de largo, con 1-2 flores sésiles; cúpula hemisférica, de (12-) 15-20 (-25) mm de diámetro, de 8-20 mm de alto, escamas de color castaño, pubescentes, adpresas, ápices agudos, engrosadas en la base; bellotas ovoides, de 15-20 mm de largo por 12-20 mm de diámetro, de color castaño, incluidas un tercio o menos de su largo en las cúpulas.

Distribución y ecología. Se le encuentra en México en los estados de Colima, Chiapas, Guerrero, Jalisco y Oaxaca, habitando en bosque de encino, bosque de pino-encino, bosque mesófilo de montaña, bosque tropical caducifolio, bosque tropical subcaducifolio, entre los 900 y 2500 m s.n.m. Florece en marzo y fructifica de junio a septiembre.

Nombres vulgares. Encino, encino asta, encino blanco, encino capulincillo, encino prieto.

Ejemplares examinados. MÉXICO. Colima: Comala, Monte de San Antonio, Monte de San Antonio, Nápoles s. n. (ENCB). Chiapas: Tenejapa, in the paraje of Oshe Wits, Breedlove 7765 (ENCB); alrededores de Tenejapa, Romero \& Rojas 4799 (IZTA). Guerrero: Chichihualco, 17 km NE de Paraíso-Pto. del Gallo, Valencia 465 (ENCB); 36 km northeast of Puerto del Gallo, Breedlove 36142 (MEXU); 16-18 km al norte de El Paraíso y Filo de Caballos, Koch 79330 (MEXU); a $2 \mathrm{~km}$ al suroeste de Tres Caminos Filo de Caballo-Puerto del Gallo, Martínez 3922 (MEXU); 8 km sobre la desviación a Pto. del Gallo-Filo de Caballo, Valencia 437 (ENCB, MEXU); 
Chilpancingo, $2 \mathrm{~km}$ al E de Omiltemi, sobre el camino a Chilpancingo, Rzedowski 16029 (ENCB); Sierra de Guerrero, Hernández 140 (MEXU); Omiltemi, 20 km al oeste de Chilpancingo, Rzedowski 15916 (MEXU); cerro Bordo del Escobal, camino Omiltemi-Chautipa, Lozano 537 (MEXU); Coyuca de Catalán, Las Palancas, Madrigal 2488 (ENCB, MEXU). Jalisco: Autlán, 20 km al sureste de Autlán, Rzedowski 14551 (ENCB); Casimiro Castillo, along trail to El Durazno, Iltis 357 (MEXU); Cuautitlán, Santana 3361, 4325 (MEXU); Talpa de Allende, Puerto El Triunfo, $19 \mathrm{~km}$ at W of Talpa de Allende road to La Cuesta and Tomatlán, Breedlove 64074, 64078, 64159, 64172 (MEXU); Zapotitlán, Cruz del Fresno, Predio de la Hda. de San Antonio, Mancera 308 (ENCB, MEXU). Oaxaca: Jilotepec, San Sebastián, MacDougall s. $n$. (MEXU); Juquila, steep mountainsides ca $80 \mathrm{~km}$ south-southwest of Sola de la Vega, McVaugh 22451 (MEXU).

Quercus uxoris se caracteriza por sus ramillas y hojas densamente pubescentes por una o más temporadas, de color dorado o castaño amarillento, ramillas gruesas (2-5 mm de diámetro); hojas maduras elípticas u ovadas, grandes (hasta de $30 \mathrm{~cm}$ de largo y $11 \mathrm{~cm}$ de ancho) y con hasta 18 dientes en cada lado.

Quercus xalapensis Humb. \& Bonpl., Plant. Aequinoct. 2: 24. Lám. 75. 1809. Tipo: México, Veracruz, Jalapa, Bonpland 4467 (holotipo en P, isotipo en B!). (Fig. 10).

Quercus candolleana Trel., Mem. Nat. Acad. Sci. 20: 191. Lám. 387. 1924. Tipo: México, Veracruz, Huatusco, Ghiesbreght 124 (holotipo en G!).

Quercus cupreata Trel. \& Müll., Bull. Torrey Bot. Club 63: 152. 1936. Tipo: México, Nuevo León, Sierra Madre Oriental: ascent to the Dos Puertos, near Hacienda Vista Hermosa, El Cercado, about 30 miles south of Monterrey, Müller 586 (holotipo en ILL, isotipo en NY!).

Quercus cupreata f. brachystachys Müll. J. Arnold Arb. 17: 176. 1936. Tipo: México, Nuevo León, mpio. de Villa Santiago, Cañón Guajuco above Villa Santiago, Müller 1327 (holotipo en A, isotipo en NY!).

Quercus cupreata f. serrata Trel. \& Müll., Bull. Torr. Bot. Club 63: 153. 1936. Tipo:

México, Nuevo León, mpio. de Villa Santiago, Potrero Redondo, Müller 595, 1933 (holotipo en ILL).

Quercus huitamalcana Trel., Mem. Nat. Acad. Sci. 20: 185. Lám. 374. 1924. Nombre basado en Q. serra Liebm., Overs. Dansk Vidensk. Selsk. Forhandl. 1854: 174. 1854. No Q. serra Unger, 1845. Tipo: México, Puebla, Huitamalco to Teziutlán, Liebmann 3560 (holotipo e isotipo en C!). 
Romero Rangel: Revisión taxonómica del complejo Acutifoliae de Quercus

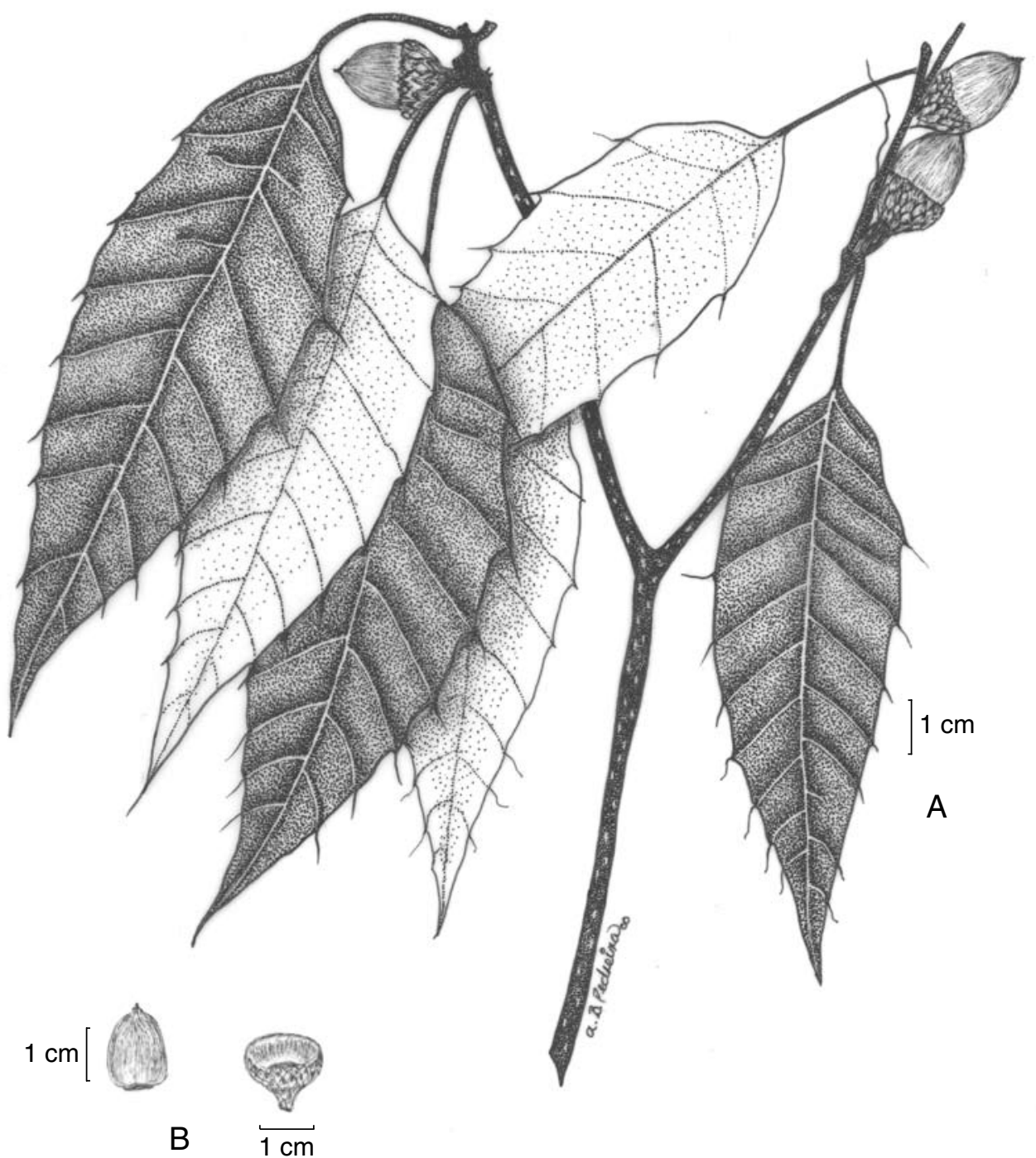

Fig. 10. Quercus xalapensis. A. rama con hojas y frutos; B. fruto y cúpula (Ventura 11623, ENCB). 
Quercus paxtalensis Müll., U.S. Dept. of Agriculture. Misc. Publ. 477: 75. 1942. Tipo: México, Chiapas, mount Paxtal (Pasitar), Matuda 1724 (holotipo en MICH, isotipo en MBG!).

Quercus runcinatifolia f. alata Trel. \& Müll., Bull. Torrey Bot. Club 63: 153. 1936. Tipo: México, Sierra Madre Oriental, Trelease \& Müller 593 (holotipo en ILL). Quercus sartorii Liebm., Overs. Dansk Vidensk. Selsk. Forhandl. 1854: 177. 1854.

Tipo: México, Veracruz, Totutla, Liebmann 3554 (holotipo en C!).

Quercus sartorii f. magna Trel., Mem. Nat. Acad. Sci. 20: 189. 1924. Tipo: México, San Bartolomé, Liebmann 3552 (holotipo en C; isotipo en MBG!).

Quercus sierramadrensis Müll., Amer. Midl. Naturalist 27(2): 478. 1942. Quercus tenuiloba f. gracilis, Müll. J. Arnold Arb. 17: 178. 1936. Tipo: México, Nuevo León, mpio. de Villa Santiago, Las Adjuntas, in the Sierra Madre Oriental, Müller 2048 (holotipo en A, isotipo en NY!).

Quercus tenuiloba Müll., J. Arnold Arb. 17: 178. 1936. México, Nuevo León, Galeana, Alamar on Hacienda Pablillo, Müller 1125 (holotipo en A, isotipo en MBG!).

Quercus tenuiloba f. hirsuta Müll., J. Arnold Arb. 17: 178. 1936. México, Nuevo León, mpio. de Villa Santiago, Potrero Redondo, Müller 2122 (holotipo en A!). Quercus vexans Trel., Mem. Nat. Acad. Sci. 20: 190-191. Lám. 386. 1924. Tipo: México, Veracruz, Huatusco, Purpus 1914 (holotipo en ILL!).

Quercus xalapensis f. jalapae Trel., Mem. Nat. Acad. Sci. 20: 193. 1924. Tipo: México, Veracruz, Jalapa, Schiede 18 (holotipo en B!).

Quercus xalapensis f. surculina Trel., Mem. Nat. Acad. Sci. 20: 193. 1924. Tipo: sin localidad, Karwinski s. $n$. (holotipo en M!).

Árbol de (4-) 8-25 (-30) m de alto y tronco de 0.15-1.50 m de diámetro; corteza oscura; ramillas de 1-3 mm de diámetro, glabrescentes, pronto glabras, rojizas, a veces muy obscuras, con lenticelas claras, menores de $1 \mathrm{~mm}$ de largo; yemas ovoides, con el ápice agudo, de 1-9 mm de largo por 1-3 mm de diámetro, con las escamas pubescentes, de color castaño; estípulas lineares, de 4-9 mm de largo por 0.5-2 mm de ancho, deciduas antes de la madurez de las hojas, con frecuencia permaneciendo en las yemas terminales; hojas jóvenes papiráceas, opacas, de color verde olivo, casi glabras en ambos lados, haz con tricomas fasciculados estipitados largos en la base de la lámina, tricomas fasciculados estipitados cortos dispersos y tricomas glandulares cerca de la nervadura primaria, envés con mechones de tricomas fasciculados estipitados largos en las axilas que forman las nervaduras secundarias, sobre la nervadura primaria, y tricomas glandulares 
abundantes, tricomas fasciculados estipitados cortos dispersos en la lámina y tricomas glandulares sobre y cerca de las nervaduras; hojas maduras coriáceas o subcoriáceas, elípticas u ovadas, de (4-) 7-18 (-20.5) cm de largo por 2-6 (-8) cm de ancho, ápice atenuado o acuminado, aristado; base aguda, obtusa, redondeada o cordada, a veces asimétrica, en hojas jóvenes o renuevos puede ser cuneada; márgenes cartilaginosos, dentados con 4-13 dientes aristados, de 1-2 mm de largo de cada lado, aristas de 1-7 mm de largo, el tamaño de los dientes disminuye hacia el ápice de la lámina, y éstos están distribuidos desde la base o desde más arriba, nervaduras secundarias 6-14 en cada lado de la lámina de la hoja, rectas o ligeramente curvas, paralelas, ascendentes, pasando directamente a los dientes y aristas; haz verde, lustroso, glabro o con algunos tricomas fasciculados estipitados cortos en la base de la nervadura primaria, nervaduras secundarias impresas o ligeramente elevadas; envés amarillento, casi glabro, con mechones de tricomas fasciculados estipitados largos en las axilas que forman las nervaduras secundarias y tricomas fasciculados estipitados cortos dispersos en la lámina y nervaduras, a veces se observan tricomas glandulares vermiformes blancos o amarillentos distribuidos en la lámina y nervaduras, epidermis lisa, papilosa, nervaduras convexas; pecíolos de (5-) 10-40 (-47) mm de largo por 0.5-1.5 (-2) mm de diámetro, glabrescentes, claros a muy oscuros; amentos masculinos de 4-9 $\mathrm{cm}$ de largo, con 30 o más flores, raquis pubescente, perianto sésil, muy pubescente, de 2-3 mm de diámetro, anteras 4-6, glabras, oblongas, de 1.5-2 mm de largo, filamentos de 1-2 $\mathrm{mm}$ de largo; amentos femeninos de 0.5-2 cm de largo, con 1-3 flores; fruto anual, solitario o en grupos de 2-3, sobre un pedúnculo de 5-20 mm de largo por 1-2 mm de diámetro; cúpula turbinada o hemisférica, de 6-20 mm de diámetro, de 4-17 mm de alto, escamas de color castaño, pubescentes, con tricomas más largos en los márgenes, adpresas, las apicales algo laxas; bellotas ovoides o globosas, de 6-20 mm de largo, por (5-) 6-15 mm de diámetro, de color castaño pálido, incluidas de un medio o menos de su largo en las cúpulas.

Distribución y ecología. Se le encuentra en México en los estados de Chiapas, Hidalgo, Nuevo León, Puebla, Querétaro, San Luis Potosí, Tamaulipas y Veracruz, habitando en bosque de encino, bosque de pino-encino y bosque mesófilo de montaña, entre $\operatorname{los} 1000$ y 2200 m s.n.m. Florece de febrero a abril y fructifica de agosto a noviembre.

Nombres vulgares. Encino blanco, encino cáscara. 
Ejemplares examinados. MÉXICO. Chiapas: Motozintla de Mendoza, southwest side of cerro Mozotal $11 \mathrm{~km}$ northwest of the junction of the road to Motozintla along the road to El Porvenir and Siltepec, Breedlove 41674 (ENCB); near Ojo de Agua, Breedlove 42675 (ENCB). Hidalgo: Actopan, Fray Francisco, 8 km al suroeste de Actopan, González 2164 (ENCB); Agua Fría, 5 km al sureste de Agua Fría, 10 km al sureste de Jacala, Müller 9473 (MEXU); Chapulhuacán, 10 km al NE de Jacala sobre la carretera a Chapulhuacán, Rzedowski 23168 (ENCB); Huehuetla, Chicontla, Turra 2064 (ENCB); Lolotla, camino de Lolotla a Ixtlahuaco, Ochoa 937 (ENCB); cerro del Toro, Vela 226 (ENCB); Molango, a 9 km del camino de Molango a Xochicatlán, Ochoa 920 (ENCB); alrededores de laguna Atezca, Romero y Rojas 4780 (IZTA); Lolotla, Romero \& Rojas 4781 (IZTA); alrededores de Chinameca, González 1600 (ENCB); Pacula, La Placita, 33 km al NE de Zimapán, Hernández 3729 (MEXU); Tenango de Doria, Tenango de Doria, Hernández 3325 (ENCB); Tlahualompa, $6 \mathrm{~km}$ al sureste de Zacualtipán, sobre el camino a Tlahualompa, Rzedowski 29460 (ENCB); proximidades de Tlahualompa, Guísar 414 (MEXU); Tlanchinol cerca de Tlanchinol, Puig 4872 (ENCB); $1 \mathrm{~km}$ al sureste de la Unidad habitacional Otongo, Rzedowski 28678(ENCB); Zacualtipán, 8 km al sur de Molango, sobre la carretera a Zacualtipán, Rzedowski 28666 (ENCB); Zacualtipán, Miranda 3277 (MEXU); Zimapán, 35 km al NE de Zimapán, Hernández 3886 (ENCB, MEXU); al NE de Zimapán, km 248 de la carretera México-Laredo, Puig 4413 (ENCB); Los Nogales, 30 km al NE de Zimapán, Hernández 6562 (ENCB). Nuevo León: Monterrey, Banco Redondo, Sierra Madre Oriental, a 30 millas de Monterrey, Müller 2237 (MEXU); Dos Puertos Vista Hermosa, Sierra Madre Oriental a 50 km S de Monterrey, Roe 1893 (MEXU); Hacienda Vista Hermosa, El Cercado, about 30 miles south of Monterrey, Müller 1326, 1332 (MEXU); Potrero Redondo, Potrero Redondo, Rayones 1893 (MEXU); Santiago, Los Cuartones, $7.5 \mathrm{~km}$ al oeste de Villa de Santiago, Valdez 945, 946 (ENCB). Puebla: Huauchinango, Hueyatlaco, Martínez 2237 (MEXU); Papatliya, Hernández 2008 (MEXU); Nanzontla, rancho Coctapehua, Colín 154 (MEXU); Necaxa, Romero y Rojas 4620 (IZTA); Necaxa, Dos Caminos, Fonseca s. n. (ENCB); Necaxa, Miranda 3049 (MEXU); Tetela de Ocampo, Talcozama, Colín 151 (ENCB); Talcozama, Simón \& Colín 151 (MEXU); Tilapa a $8 \mathrm{~km}$ al sur de Tetela de Ocampo, Vázquez 80 (MEXU); Teziutlán, El Carrizal, 3 km al NE de Teziutlán, Pineda 651a (ENCB); Villa Juárez, km 8.0 del camino que baja a la mina El Paraíso, Sarukhán 986, 1104 (MEXU); Villa JuárezParaiso, Miranda 3185 (MEXU); Xicotepec de Juárez, 5 km al suroeste de Villa de Juárez, González 170, 285 (ENCB); Zacapoaxtla, Apulco, Rzedowski 31754 (ENCB); Huipulco, Rosales 2124 (MEXU). Querétaro: Landa, Tilaco, Puig 3618 
(ENCB); Jalpan, Tancoyol, El Carrizal, Puig 4484 (ENCB); La Parada, Puig 4507 (ENCB). San Luis Potosí: Cerritos, cerro Prieto, Rivera 2231 (MEXU); Ciudad del Maíz, a 20 min. de Ciudad del Maíz, Manning 53445 (MEXU); 0.5 km NE de las Abritas, Rzedowski 11127 (ENCB); 39 km (by road) NE of Ciudad del Maíz on Hwy 80 at km 223, Roe 2307 (MEXU); km 253 carretera San Luis Potosí-Antiguo Morelos, Rzedowski 8380 (ENCB); Las Abritas, Puig 3421 (ENCB); mountains about 10 miles northeast of Ciudad del Maíz, McVaugh 10429 (MEXU); Rayón, 15 km al E de Rayón sobre la carretera a Ciudad Valles, Rzedowski 24455 (ENCB); Zaragoza, Los Hoyos, km 51 carretera San Luis Potosí-Ríoverde, Rzedowski 9361 (ENCB); Ríoverde, camino hacia las minas de San Isidro, ejido Milpitas, Ballin s. $n$. (MEXU); km 58, carretera San Luis Potosí-Ríoverde Rzedowski 8068 (ENCB); San Nicolás Tolentino, cerca de Cruz de Encino, Rzedowski 11316 (ENCB); Zaragoza, a $18 \mathrm{~km}$ al oeste de Santa Catarina, sobre la carretera a San Luis Potosí, Puig 3665 (ENCB); Tamasopo, $5 \mathrm{~km}$ al sureste de Tamasopo, Rzedowski 6927 (MEXU); 7 km al norte del ejido Damián Carmona, Rzedowski 10628 (ENCB); Tamasopo, Puig 3645 (ENCB); Tamazunchale, entre Tamán y San Francisco, Rzedowski 1107a (IEB); Tamasopo, Ejido el Palmar, Flamand 8 (ENCB); Xilitla, $3 \mathrm{~km}$ al noroeste de Xilitla, Rzedowski 7249 (ENCB); Xilitla, 5 km al norte de Soledad de Zaragoza, Rzedowski 7185, 7186 (MEXU); 5 km al noroeste de Ahuacatlán, Rzedowski 9275 (MEXU); El Balcón, cerca de Ahuacatlán, Rzedowski 9243 (ENCB); Potrerillos, Rzedowski 10010 (ENCB). Tamaulipas: Aldama, 56 km al norte de Manuel, Sierra de Tamaulipas, González-Medrano 12468 (MEXU); Gómez Farías, Sierra de Guatemala, vicinity of Rancho del Cielo Biological Station, Sullivan 292, 328, 663 (ENCB); from Rancho del Cielo to Ojo de Agua del Indio, Hernández 5941, 5951 (MEXU); Rancho del Cielo a 11 km al norte de Gómez Farías, González-Medrano 7401, 7420 (MEXU); Rancho del Cielo, Hernández 5820 (MEXU); Rancho del Cielo, Sierra de Guatemala, above Gómez Farías, Hernández 52038, 52189 (MEXU); Sierra de Guatemala, Sullivan 522 (ENCB); vicinity of Rancho del Cielo, Biological Southmost Collage Station of Texas, Sullivan 429 (ENCB); Guemes, $1 \mathrm{~km}$ al sur de la Yerbabuena, González-Medrano, 17538 (MEXU); Hidalgo, 58-59 km al oeste de Barreta, González 6367 (MEXU); 58-59 km al oeste de Barretal, González 6372 (MEXU); $60 \mathrm{~km}$ al oeste de Barretal, González 6387 (MEXU); Jaumave, entre Joya del Pinal y Joya de Salas, Martínez 3742 (MEXU); La Reja, Castañeda 2760 (ENCB); La Reja, Castañeda 2747, 2756 (MEXU); Manuel González, 4 km al norte de San Andrés, González-Medrano 7186 (MEXU); $56 \mathrm{~km}$ al norte de Manuel, González 12468 (MEXU); Ocampo, $32 \mathrm{~km}$ al E de Tula, along road to Ocampo, Breedlove 63673 (MEXU). Veracruz: Atzalan, 4 km al NE de Altotonga, Hansen 
18729 (MEXU); Banderilla, 2 km al NE de Banderilla, rancho La Mesa, Zola 652 (MEXU); Coatepec, Briones, Estrada 770 (ENCB); Córdoba, Córdoba-Huatusco, Barranca del Monte Salas, Chiang 149 (ENCB); Coscomatepec, cañada junto al río Jamapa, camino de Calcahualco a Coscomatepec, Lot 1182 (ENCB); Huatusco, 4 km al sur de Huatusco, Rzedowski 32946 (ENCB); 10 km NE del Mirador, NE de Huatusco, Rzedowski 23167 (ENCB); 2 km al oeste de Río Seco, carretera HuatuscoCoscomatepec, Avendaño 796 (ENCB); 6 km NE del Mirador, NE de Huatusco, Müller 9360 (MEXU); Puente sobre el río Jamapa, 15 km al suroeste de Huatusco, Rzedowski 19053 (ENCB); Tlaltongo, $10 \mathrm{~km}$ al suroeste de Huatusco, Rzedowski 18938, 18931 (ENCB); Ixhuacán de los Reyes, 6 km (by road) ESE of Ixhuacán de los Reyes, Nee 22475 (MEXU); Jilotepec, El Zacatal, Ventura 16976 (MEXU); El Esquilón, Gutiérrez 2726 (MEXU); Esquilón, carretera Jalapa-Naolinco, Dorantes 1762 (ENCB); Esquilón, Ventura 7117 (ENCB); Naolinco, Naolinco, Ventura 8919, (ENCB); Orizaba, cerro de San Cristóbal, 5 km al suroeste de Orizaba, Rzedowski 12187 (ENCB); Soteapan, 1 km al oeste de San Fernando, Bye 7549 (MEXU); Ocotal Chico, 20 km al norte de San Pedro Soteapan, Guevara 15 (MEXU); Xalapa, hills about Xalapa, Pringle 8108 (MEXU); al sur de Xalapa, Zola 808 (MEXU); camino al sumidero, Zola 748 (MEXU); Jardín Botánico Clavijero, $3 \mathrm{~km}$ suroeste del centro de Xalapa, Nee 33068 (MEXU); Jardín Botánico Rancho Guadalupe a 3 km, Vovides 00060, 00062 (ENCB, MEXU); Lomas El Mirador, Ventura 2361 (ENCB); Palenque, Zola 770 (MEXU); Xico, Texolo, Ventura 11623 (ENCB).

Quercus xalapensis se caracteriza por sus hojas maduras elípticas u ovadas, de regular tamaño, márgenes dentados con 4-13 dientes aristados de cada lado; epidermis del envés lisa y papilosa, con escasos mechones de tricomas fasciculados estipitados largos en las axilas que forman las nervaduras secundarias y tricomas fasciculados estipitados cortos muy dispersos en la lámina y nervaduras. Se hicieron observaciones de la arquitectura foliar de ejemplares de los estados de Puebla, San Luis Potosí y Tamaulipas (Romero R., 2001), encontrándose que la especie tiene la vena primaria moderadamente gruesa, venas secundarias con tres ramas formando ángulos de divergencia que disminuyen hacia el ápice, venas intersecundarias distribuidas en la base y en el ápice de la hoja, aréolas de forma cuadrangular y vénulas predominantemente ramificadas.

Por otro lado, se realizaron observaciones de las poblaciones en Lolotla y Molango en Hidalgo, Necaxa en Puebla y Xalapa en Veracruz, debido a que de estas localidades existe material identificado como Q. sartorii y Q. xalapensis. Sin embargo, no se encontraron diferencias claras que pudieran indicar que se trata 
de especies distintas. Romero R. (2001) hizo un estudio numérico con ejemplares identificados con estos nombres, hallando que comparten caracteres cualitativos y cuantitativos de hoja y fruto, sin observar formación de grupos que indiquen que se trata de dos taxa.

La revisión de los tipos de Q. xalapensis (Bonpland 4467, B) y Q. sartorii (Liebmann 3554, C) y las descripciones originales mostró que Q. sartorii es sinónimo de $Q$. xalapensis. Además, las localidades tipo de estas dos especies se encuentran en el estado de Veracruz, entidad donde se han colectado bastantes ejemplares e identificado con estos dos nombres, lo que favoreció el estudio de una cantidad importante de material de herbario.

También se revisaron los tipos y las descripciones originales de $Q$. candolleana Trel., Q. huitamalcana Trel., Q. xalapensis f. jalapae Trel., Q. xalapensis f. surculina Trel., Q. sartorii Liebm., Q. sartorii f. magma Liebm., Q. cupreata Trel. \& Müll., Q. runcinatifolia f. alata Trel. \& Müll., Q. cupreata f. brachystachys Müll., Q. cupreata f. serrata Trel. \& Müll., Q. tenuiloba Müll., Q. tenuiloba f. hirsuta Müll., Q. vexans Trel., Q. paxtalensis Müll. y Q. sierramadrensis Müll; en todos ellos se encontraron los caracteres que distinguen a $Q$. xalapensis, por lo que se considera que representan variaciones de esta misma y en consecuencia corresponden a sinónimos (Cuadro 1).

\section{CONSIDERACIONES FINALES}

El complejo Acutifoliae se ha considerado un grupo difícil en su taxonomía, debido a la riqueza de nombres involucrados y a la gran variabilidad morfológica relacionada con factores ambientales, daños ocasionados a las ramas y la edad de los individuos. Tal circunstancia ha provocado confusiones en la delimitación de las especies, haciéndose necesario el estudio de las poblaciones en su hábitat, procedimiento que fue de trascendental importancia en la definición de los resultados de esta revisión. La búsqueda de más caracteres diagnósticos, sobre todo en los aspectos de arquitectura foliar y morfología de las estructuras reproductoras (Romero R. et al. 2000b), muy probablemente contribuirá a afinar la presente aproximación. Aunque se ha invocado a la hibridación como causa de variabilidad morfológica de los encinos, ésta no siempre es fácil identificar en el caso del grupo estudiado y será necesario documentarla con trabajos de biología molecular.

México es el país que posee el mayor número de especies del género Quercus; sin embargo, muchos de los bosques en donde habitan estas plantas ya han desaparecido, mientras que otros presentan diferentes grados de deterioro. En 
este contexto cabe destacar el hecho que del complejo Acutifoliae Quercus cortesii, $Q$. brenesii y $Q$. furfuracea deben considerarse como amenazadas, debido a su distribución restringida y a que sus individuos son escasos.

\section{AGRADECIMIENTOS}

Este trabajo es parte de la tesis doctoral de la autora, la cual tuvo el apoyo del Consejo Nacional de Ciencia y Tecnología (CONACyT-95882). Se agradece de manera muy especial al M. en C. Carlos Rojas Zenteno por su participación en el trabajo de campo y en la elaboración de las ilustraciones, también a la bióloga Adriana Bernal Pedreira por la preparación de los dibujos. De manera importante y con gran aprecio se agradece a la bióloga Lourdes Aguilar Enríquez por sus valiosas observaciones.

\section{LITERATURA CITADA}

Camus, A. 1938. Les chênes. Monographie du genre Quercus. Paul Lechevalier, Paris. 373 pp.

González Villarreal, L. M. 1986. Contribución al conocimiento del género Quercus (Fagaceae) en el estado de Jalisco. Colección Flora de Jalisco. Instituto de Botánica, Universidad Autónoma de Guadalajara. Guadalajara, Jalisco. 240 pp.

Hickey, L. 1973. Classification of the architecture of dicotyledonous leaves. Am. J. Bot. 60: 17-33.

Jones, J. 1986. Evolution of the Fagaceae: the implications of foliar features. Ann. Missouri Bot. Gard. 73: 228-275.

Martínez, M. 1954. Los encinos del Estado de México. Comisión Botánica Exploradora del Estado de México. Gobierno del Estado de México. Dirección de Agricultura y Ganadería. Toluca, México. 77 pp.

Müller, C. H. 1936a. New and noteworthy trees in Texas and Mexico. Bull. Torrey Bot. Club 63: $147-155$.

Müller, C. H. 1936b. Studies in the oaks of the mountains of northeastern Mexico. J. Arnold Arb. 16: 174-179.

Müller, C. H. 1942a. The Central American species of Quercus. U.S. Dept. Agr. Misc. Publ. 477: 1-92. lám. 1-124.

Müller, C. H. 1942b. Notes on the American flora, chiefly Mexican. Am. Midl. Nat. 27(2): 470-490.

Müller, C. H. 1951. The oaks of Texas. Contr. Texas Res. Found. 1: 21-323. 
Müller, C. H. \& R. McVaugh. 1972. The oaks (Quercus) described by Née (1801), and by Humboldt \& Bonpland (1809), with coments on related species. Contr. Univ. Mich. Herb. 9(7): 507-522.

Nixon, K. 1993. Infrageneric classification of Quercus (Fagaceae) and typification of sectional names. Ann. Sci. Forest. 50: 25-34.

Romero R., S., R. Lira S. y P. Dávila. 2000a. A phenetic study of the taxonomic delimitation of Quercus acutifolia and Q. conspersa (Fagaceae). Brittonia 52(2): 177-187.

Romero R., S., C. Rojas Z. y S. Gómez. 2000b. Flores hermafroditas de Quercus glaucoides (Fagaceae) en el estado de Michoacán, México. Acta Bot. Mex. 52: 49-54.

Romero R., S., 2001. Estudio taxonómico de la serie Acutifoliae (Quercus, Fagaceae). Tesis doctoral. Facultad de Ciencias, Universidad Nacional Autónoma de México. México, D.F. 174 pp.

Trelease, W. 1924. The American oaks. Mem. Nat. Acad. Sci. 20:1-255. Lám. 1-420.

Recibido en noviembre de 2002.

Aceptado en abril de 2006. 